\title{
1 Time-resolved parameterization of aperiodic and periodic brain activity
}

3 Luc E. Wilson ${ }^{1}$, Jason da Silva Castanheira ${ }^{1}$, Sylvain Baillet ${ }^{1} \uparrow$

$4{ }^{1}$ McConnell Brain Imaging Centre, Montreal Neurological Institute, McGill University, $5 \quad$ Montreal, QC H3A 2B4, Canada

6 † Corresponding authors: sylvain.baillet@mcgill.ca.

8 Abstract

9 Macroscopic neural dynamics comprise both aperiodic and periodic components. Recent advances

10 in parameterizing neural power spectra offer practical tools for evaluating these features

11 separately. Although neural signals vary dynamically and express non-stationarity in relation to 12 ongoing behaviour and perception, current methods yield static spectral decompositions. Here, we

13 introduce Spectral Parameterization Resolved in Time (SPRiNT) as a novel method for 14 decomposing complex neural dynamics into periodic and aperiodic spectral elements in a time-

15 resolved manner. First, we demonstrate, with naturalistic synthetic data, SPRiNT's capacity to 16 reliably recover time-varying spectral features and emphasize SPRiNT's specific strengths over 17 other time-frequency parameterization approaches based on wavelets. Second, we use SPRiNT to 18 further illustrate how aperiodic spectral features fluctuate across time in resting-state 19 electroencephalography $(n=178)$ and relate changes in aperiodic parameters to participants'

20 demographics and behaviour. Lastly, we use SPRiNT to demonstrate aperiodic dynamics related

21 to movement behaviour in intracranial recordings in rodents. We foresee SPRiNT responding to

22 growing neuroscientific interests in the parameterization of time-varying neural power spectra and

23 advancing the quantitation of complex neural dynamics at the natural time scales of behaviour. 
24 Keywords: aperiodic, periodic, time-resolved, parameterization, power spectra, neural

25 oscillations, electrophysiology.

27 Significance Statement

The new method and reported findings address a growing interest in neuroscience for

29 research tools that can reliably decompose brain activity at the mesoscopic scale into interpretable

30 components. We show that the new approach proposed herein is capable of tracking transient,

31 dynamic spectral (aperiodic and periodic) components across time, both in synthetic simulated and

32 experimental data. We anticipate that this novel technique, SPRiNT, will enable new neuroscience

33 inquiries that reconcile multifaceted neural dynamics with complex behaviour.

34

35

36

37

38

39

40

41

42

43

44

45

46 


\section{Introduction}

48 The brain constantly expresses a repertoire of complex dynamics related to behaviour in health

49 and disease. Neural oscillations, for instance, are rhythmic components of brain activity that

50 emerge from a background of aperiodic fluctuations recorded with a range of electrophysiological

51 techniques at the mesoscopic scale (Buzsáki, 2006). Brain oscillations and their dynamics have

52 been causally linked to individual behaviour and cognition (Albouy et al., 2017), and shape brain

53 responses to sensory stimulation (Samaha et al., 2020).

Current methods for measuring the time-varying properties of neural fluctuations include several time-frequency decomposition techniques such as Hilbert, wavelet, and short-time Fourier signal transforms (Bruns, 2004; Cohen, 2014). Following spectral decomposition, oscillatory

57 activity within the empirical bands of electrophysiology manifests as peaks of signal power

58 (Buzsáki \& Watson, 2012; Cohen, 2014). However, time-resolved signal power decompositions

59 do not explicitly account for the presence of aperiodic signal components, which challenge both

60 the detection and the interpretability of spectral peaks as genuine periodic signal elements

61 (Donoghue et al., 2020). This is critical as aperiodic and periodic components of neural activity

62 represent distinct, although possibly interdependent physiological mechanisms (Gao et al., 2017).

Aperiodic neural activity is characterized by a reciprocal distribution of power with

64 frequency (1/f), which can be parameterized with two scalars: exponent and offset. These

65 parameters are physiologically meaningful: current constructs consider the offset as reflecting

66 neuronal population spiking, and the exponent as related to the integration of synaptic currents

67 (Voytek \& Knight, 2015) and reflecting the balance between excitatory (E) and inhibitory (I)

68 currents (i.e., the larger the exponent, the stronger the inhibition; Chini et al., 2021; Gao et al.,

69 2017; Waschke et al., 2021). Aperiodic neural activity is ubiquitous throughout the brain (He, 
2014); it differentiates healthy aging (Cellier et al., 2021; Donoghue et al., 2020; Hill et al., 2022;

71 Ostlund et al., 2022; Schaworonkow \& Voytek, 2021; Voytek et al., 2015), and is proposed as a

72 potential marker of neuropsychiatric conditions (Molina et al., 2020) and epilepsy (van Heumen

73 et al., 2021). Though the study of aperiodic neural activity has recently advanced, unanswered

74 questions remain about its functional relevance, which requires an expanded toolkit to track their

75 evolution across time and the broadest possible expressions of behaviour.

One little studied aspect of aperiodic activity is its fluctuations, both spontaneously over time, and with task and mental states. Baseline normalization is a common approach to compensate

78 for aperiodic contributions to time-frequency decompositions (Cohen, 2014), with the underlying assumption, however, that characteristics of aperiodic activity (exponent and offset) remain unchanged throughout the data length — an assumption that is challenged by recent empirical data

81 (Van Heumen et al., 2021; Waschke et al., 2021). Akin to the motivations behind

82 aperiodic/periodic spectral parameterization and signal decomposition techniques (Donoghue et

83 al., 2020, Wen \& Liu, 2016), undetected temporal variations of the neural spectrogram may

84 conflate fluctuations in aperiodic activity with modulations of oscillatory signals, hence distorting

85 data interpretation (Donoghue et al., 2020). Recent methodological advances have contributed

86 practical tools to decompose and parameterize the neural power spectrum into aperiodic (scale-

87 free, 1/f) and narrow-band periodic components (spectral peaks of certain widths; Donoghue et al.,

88 2020; Wen \& Liu, 2016; He, 2014). One such practical tool (specparam) sequentially fits aperiodic

89 and oscillatory parametric components to the empirical neural power spectrum (Donoghue et al.,

90 2020). The resulting model for the aperiodic component is represented with exponent and offset

91 scalar parameters, and periodic elements are modelled with a series of Gaussian functions

92 characterised with three scalar parameters (centre frequency, amplitude, and standard deviation). 
93 Specparam accounts for static spectral representations and has yet to be extended to account for

94 the non-stationary contents of neural time series.

95 We introduce SPRiNT (Spectral Parameterization Resolved in Time) as a novel approach

96 to identify and model dynamic shifts in aperiodic and periodic brain activity, yielding a time-

97 resolved parameterization of empirical neural spectrograms. We validate the method with an

98 extensive set of naturalistic simulations of neural data, with benchmark comparisons to

99 parameterized wavelet signal decompositions. Using SPRiNT, we also show that aperiodic

100 fluctuations of the spectrogram can be related to meaningful behavioural and demographic

101 variables from human EEG resting-state data and electrophysiological recordings from free-

102 moving rodents.

103

104 Results

105 SPRiNT consists of the following methodological steps. First, short-time Fourier transforms

106 (STFT) are derived from overlapping time windows that slide over data time series. Second, the

107 resulting STFT coefficients are averaged over consecutive time windows to produce a smooth

108 estimate of the power spectral density of the recorded data. Third, the resulting spectrum is

109 parameterized into aperiodic and periodic components using specparam (see Methods). As the

110 procedure is repeated over the entire length of the data, SPRiNT produces a time-resolved

111 parameterization of the data's spectrogram (Figure 1A). The resulting parameters can then be

112 compiled into time-frequency representations for visualization and further derivations. A fourth

113 step consists of an optional post-processing procedure meant to prune outlier transient

114 manifestations of periodic signal components (Figure 1 - figure supplement 1). 
a) overview
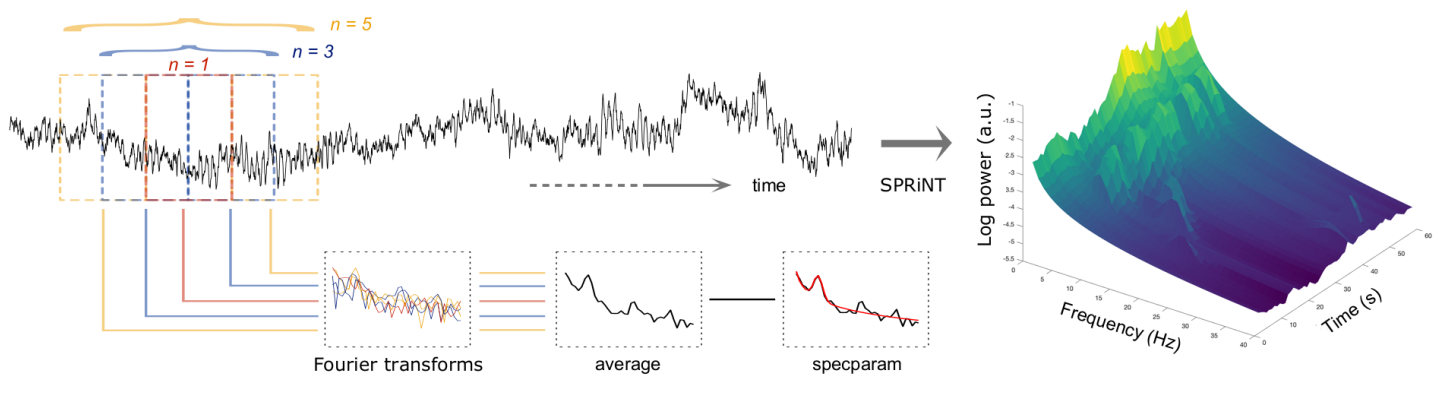

b) simulation I: accuracy of time-resolved parameterization

c) simulation II: generalization of time-resolved parameterization

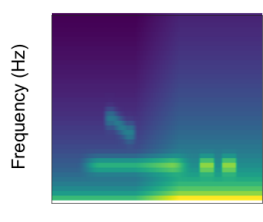

Time (s)

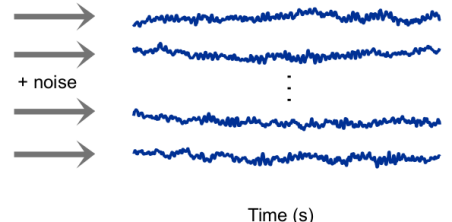

Time (s)
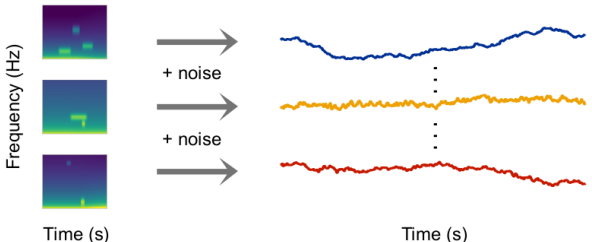

Figure 1: Methods Synopsis

118 (A) Overview of the approach implemented in SPRiNT. At each time bin along a neural time

119 series (black trace) $n$ overlapping time windows are Fourier-transformed to yield an estimate of

120 spectral contents, which is subsequently parameterized using specparam (Donoghue et al.,

121 2020). The procedure is replicated across time over sliding windows to generate a parameterized

122 spectrogram of neural activity. (B) Simulation challenge I. We simulated 10,000 time-series

123 composed of time-varying spectral (aperiodic and periodic) features. These ground-truth time

124 series were used to evaluate the respective performances of SPRiNT and those from a wavelet-

125 specparam alternative. (C) Simulation challenge II. We simulated another 10,000 time series

126 each composed of different time-varying spectral (aperiodic and periodic) ground-truth features.

127 These time series were used to assess SPRiNT's ability to detect these features.

128 Figure supplement 1. Overview of the outlier peak removal process.

We generated a total of 20,000 naturalistic synthetic neural time series with non-stationary 
et al., 2019) with MATLAB (R2020a; Natick, MA). We first tested SPRiNT's ability to detect and

133 track transient and chirping periodic elements (with time-changing aperiodic components) and

134 benchmarked its performance against parametrized wavelet signal decompositions (Figure 1B). A

135 second validation challenge focused on simulations derived from randomly generated sets of

136 realistic aperiodic and periodic parameters; this challenge served to assess SPRiNT's performance

137 across naturalistic heterogenous signals (Figure 1C; see Methods). Further below, we describe the 138 application of SPRiNT to a variety of empirical data from human and rodent electrophysiology.

\section{Methods benchmarking (synthetic data).}

141 We first simulated 10,000 time series (60 s duration) with aperiodic components transitioning

142 linearly between $\mathrm{t}=24 \mathrm{~s}$ and $\mathrm{t}=36 \mathrm{~s}$, from an initial exponent of $1.5 \mathrm{~Hz}$ and offset of -2.56 [arbitrary

143 units, a.u.] to a final exponent of $2.0 \mathrm{~Hz}$ and offset of -1.41 a.u. The periodic components of the

144 simulated signals included transient activity in the alpha band (centre frequency: $8 \mathrm{~Hz}$; amplitude:

145 1.2 a.u.; standard deviation: $1.2 \mathrm{~Hz}$ ) occurring between 8-40 s, 41-46 s, and 47-52 s, and a down-

146 chirping oscillation in the beta band (centre frequency decreasing from 18 to $15 \mathrm{~Hz}$; amplitude:

$147 \quad 0.9$ a.u.; standard deviation: $1.4 \mathrm{~Hz}$, between 15-25 s (Figure 1B). We applied SPRiNT on each

148 simulated time series, post-processed the resulting parameter estimates to remove outlier transient

149 periodic components, and derived goodness-of-fit statistics of the SPRiNT parameter estimates

150 with their ground-truth values. We compared SPRiNT's performances to the parameterization of

151 temporally smoothed spectrograms obtained from Morlet wavelets time-frequency

152 decompositions of the simulated time series (smoothed using a 4 s Gaussian kernel, standard

153 deviation $=1 \mathrm{~s}$ ). We refer to this approach as wavelet-specparam (see Methods). We assessed the

154 respective performances of SPRiNT and wavelet-specparam with measures of Mean Absolute 
155 Error (MAE) on their respective estimates of spectrogram time-frequency profiles and of the

156 parameters of their a/periodic components across time.

a) spectrogram models
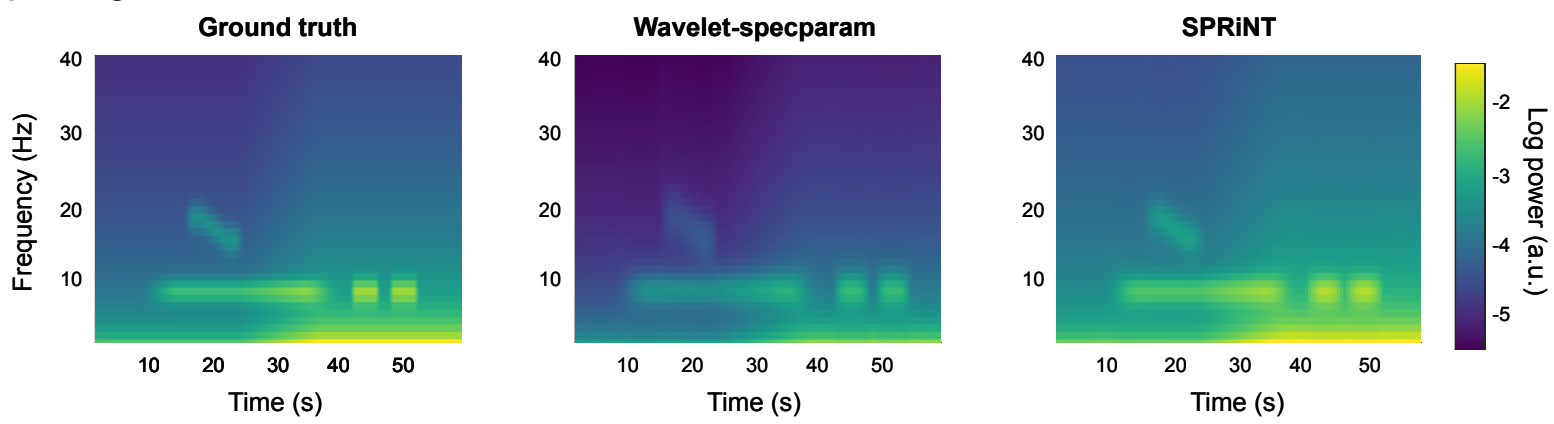

b) aperiodic parameter fits
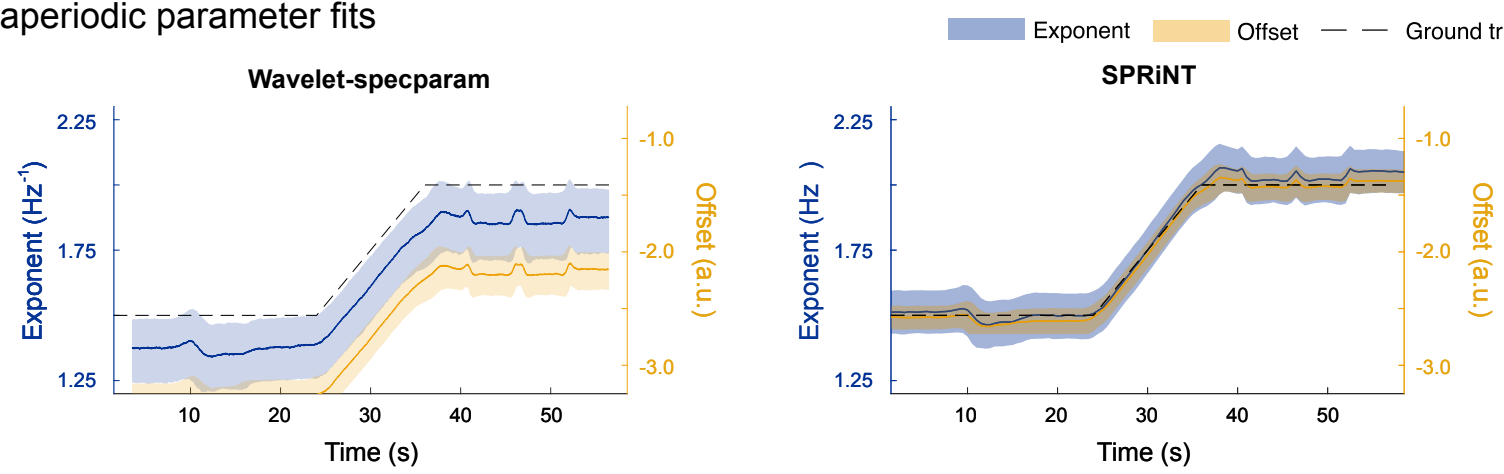

c) periodic parameter fits
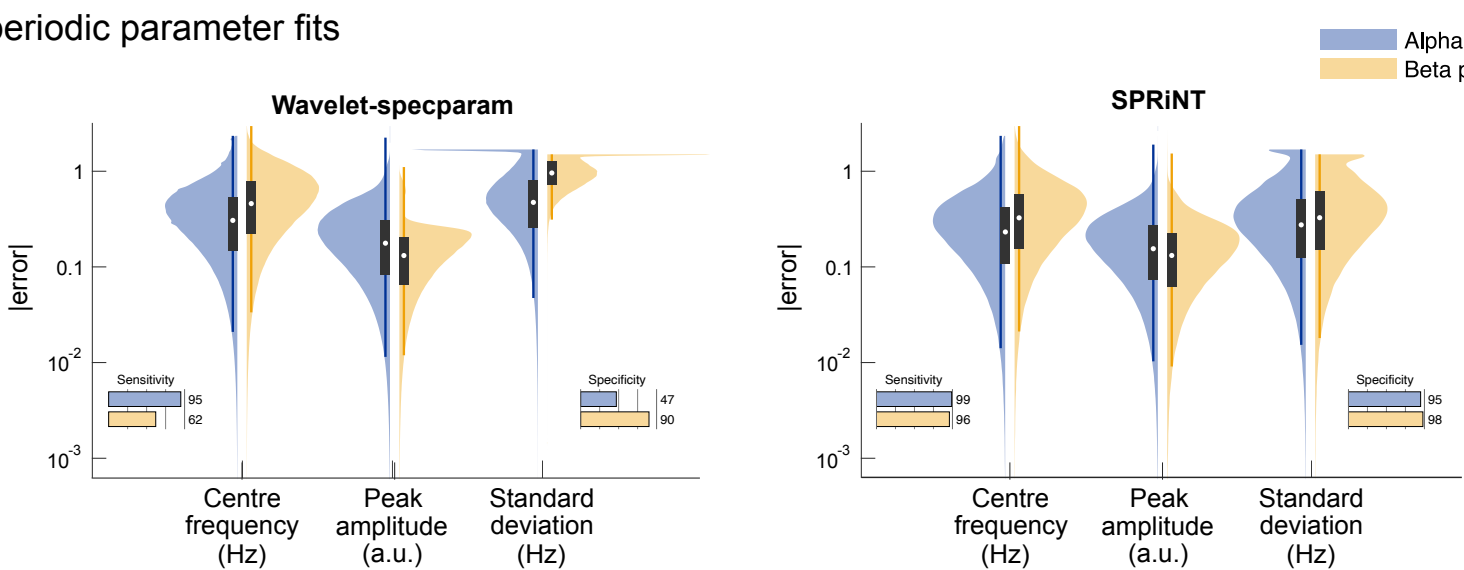

159 Figure 2: SPRiNT vs. wavelet-specparam performances (Simulation Challenge I).

160 (A) Ground truth spectrogram (left) and averaged modelled spectrograms from the wavelet-

161 specparam approach (middle) and SPRiNT (right; $n=10,000)$. (B) Aperiodic parameter

162 estimates (lines: median; shaded regions: first and third quartiles, $n=10,000$ ) across time from 
163 wavelet-specparam (left) and SPRiNT (right; black: ground truth; blue: exponent; yellow:

164 offset). (C) Absolute error (and detection performance) of alpha and beta-band rhythmic

165 components for wavelet-specparam (left) and SPRiNT (right). Violin plots represent the sample

166 distributions ( $n=10,000$; blue: alpha peak; yellow: beta peak; white circle: median, grey box:

167 first and third quartiles; whiskers: range).

168 Figure supplement 1. Periodic parameter estimates across time ( $n=10,000$ simulations). aperiodic spectrogram: wavelet-specparam $\mathrm{MAE}=0.60, \mathrm{SEM}=6.7 \times 10^{-6} ; \mathrm{SPRiNT} \mathrm{MAE}=0.06$,

$\left.175 \mathrm{SEM}=4.0 \times 10^{-5}\right)$. The performances of the two methods in parametrizing periodic components were strong and similar (wavelet-specparam $\mathrm{MAE}=0.05, \mathrm{SEM}=4.0 \times 10^{-6} ; \mathrm{SPRiNT} \mathrm{MAE}=0.03$, $\left.177 \quad \mathrm{SEM}=2.7 \times 10^{-5}\right)$. $\mathrm{SEM}=1.1 \times 10^{-4}$ ) were substantially less than those from wavelet-specparam (exponent MAE = $0.19, \mathrm{SEM}=1.5 \times 10^{-5} ;$ offset $\mathrm{MAE}=0.78, \mathrm{SEM}=2.6 \times 10^{-5} ;$ Figure $\left.2 \mathrm{~A}\right)$. SPRiNT detected periodic

181 alpha activity with higher sensitivity (99\% at time bins with maximum peak amplitude) and 182 specificity (96\%) than wavelet-specparam (95\% sensitivity, 47\% specificity). SPRiNT estimates 183 of alpha peak parameters were also closer to ground truth (centre frequency, amplitude, bandwidth $\operatorname{MAE}[\mathrm{SEM}]=0.33\left[3.6 \times 10^{-4}\right] \mathrm{Hz}, 0.20\left[1.7 \times 10^{-4}\right]$ a.u., $0.42\left[4.8 \times 10^{-4}\right] \mathrm{Hz}$, respectively) than 
186

187

188

189

190

191

192

193

194

195

196

197

198

199

200

201

202

203

204

205

206

207

208

respectively; Figure 2C). SPRiNT detected and tracked down-chirping beta periodic activity with higher sensitivity (95\% at time bins with maximum peak amplitude) and specificity (98\%) than wavelet-specparam (62\% sensitivity, 90\% specificity). SPRiNT's estimates of beta peak parameters were also closer to ground truth (centre frequency, amplitude, bandwidth MAE $=0.43$ $\left[9.4 \times 10^{-4}\right] \mathrm{Hz}, 0.17\left[3.6 \times 10^{-4}\right]$ a.u., $0.48\left[1.1 \times 10^{-3}\right] \mathrm{Hz}$, respectively) than with wavelet-specparam (centre frequency, amplitude, bandwidth $\mathrm{MAE}=0.58\left[1.4 \times 10^{-4}\right] \mathrm{Hz}, 0.16\left[4.2 \times 10^{-5}\right]$ a.u., 1.05 $\left[1.2 \times 10^{-4}\right] \mathrm{Hz}$, respectively; Figure $\left.2 \mathrm{C}\right)$. We noted that both methods tended to overestimate peak bandwidths (Figure 2 - figure supplement 1), and the effect was more pronounced with waveletspecparam (Figure 2C). We report raw SPRiNT performances prior to the removal of outlier peaks in Supplemental Materials.

\section{Generalization of SPRiNT across generic aperiodic and periodic fluctuations (synthetic}

data).

We simulated 10,000 additional time series consisting of aperiodic and periodic components, whose parameters were sampled continuously from realistic ranges (Figure 1C). The generators of each trial time series comprised i) one aperiodic component whose exponent and offset parameters were shifted linearly over time, and ii) 0 to 4 periodic components (see Methods for details). SPRiNT, followed by outlier peak post-processing, recovered $69 \%$ of the simulated periodic components, with $89 \%$ specificity $(70 \%$ sensitivity and $73 \%$ specificity prior to outlier removal as shown in Figure 3 - figure supplement 1). Aperiodic exponent and offset parameters were recovered with MAEs of 0.12 and 0.15 , respectively. The centre frequency, amplitude, and frequency width of periodic components were recovered with MAEs of 0.45, 0.23, and 0.49, respectively (Figure 3B). We evaluated whether the detection and accuracy of parameter estimates 
209 of periodic components depended on their frequency and amplitude (Figure 3C). The synthetized

210 data showed that overall, SPRiNT accurately detects up to two simultaneous periodic components

211 (Figure 3D). We also found that periodic components of lower frequencies were more challenging

212 to detect (Figure 3C \& 3D) because their peak spectral component tended to be masked by the

213 aperiodic $(1 / \mathrm{f})$ component of the power spectrum. Model fit errors $(\mathrm{MAE}=0.032)$ varied

214 significantly with the number of simultaneous periodic components, but this effect was small $(\beta=$

$215-0.0001, \mathrm{SE}=6.7 \times 10^{-6}, 95 \%$ CI $[-0.00010 .0001] ; \mathrm{R}^{2}=0.0003 ;$ Figure 3E). 
bioRxiv preprint doi: https://doi.org/10.1101/2022.01.21.477243; this version posted February 9, 2022. The copyright holder for this preprint (which was not certified by peer review) is the author/funder, who has granted bioRxiv a license to display the preprint in perpetuity. It is made available under aCC-BY-NC-ND 4.0 International license.

a) SPRiNT output example

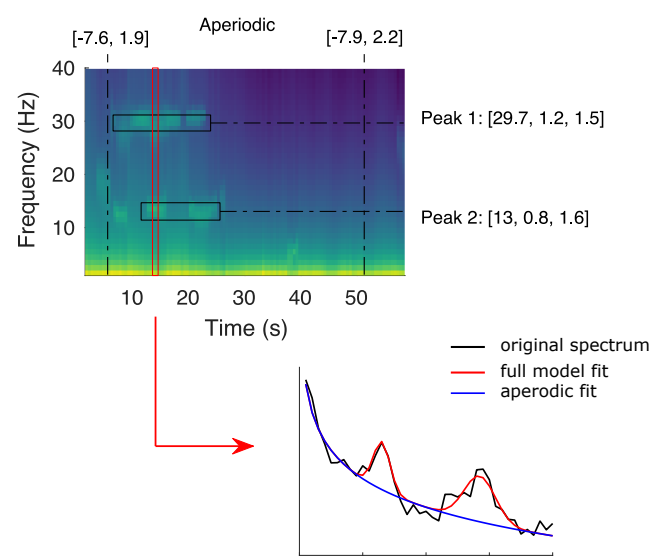

c) peak-fitting probability
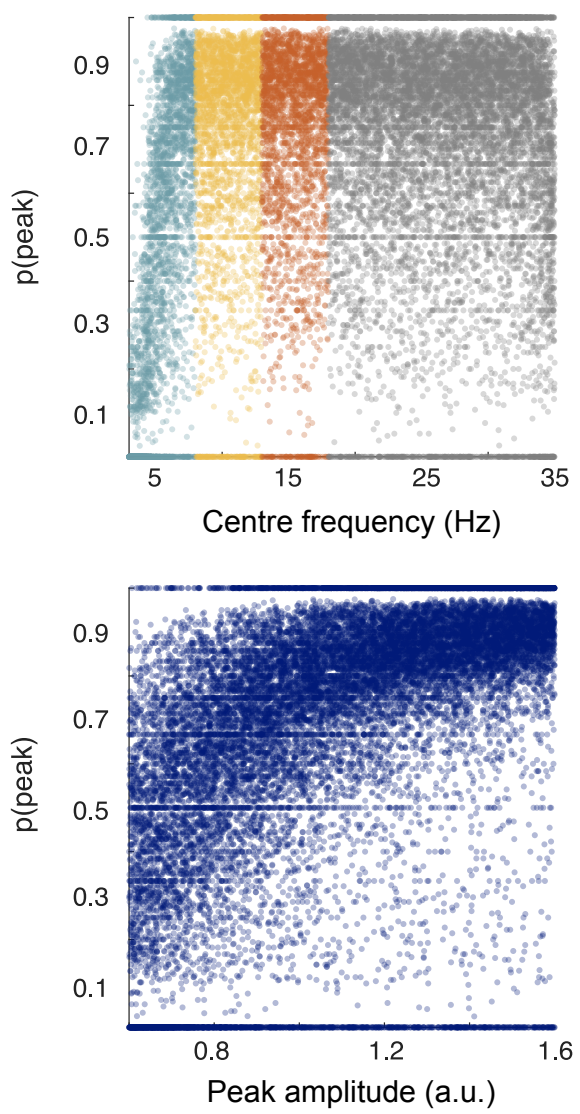

b) SPRiNT parameter errors

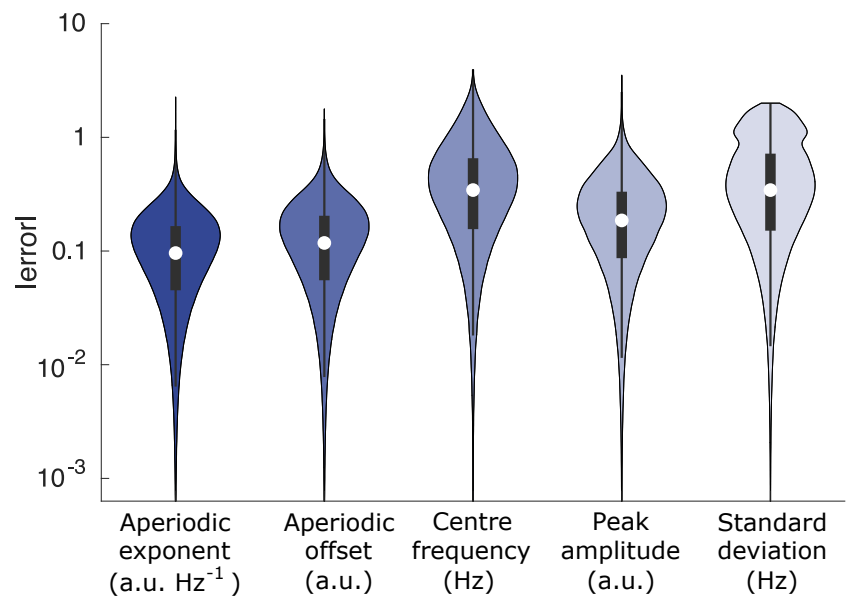

d) fitted peaks vs. actual peaks

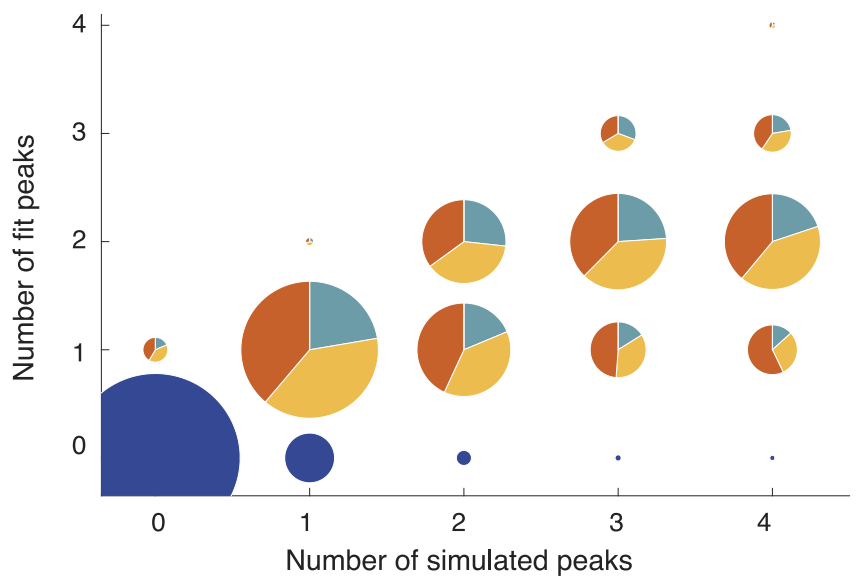

e) number of peaks does not affect model fit error

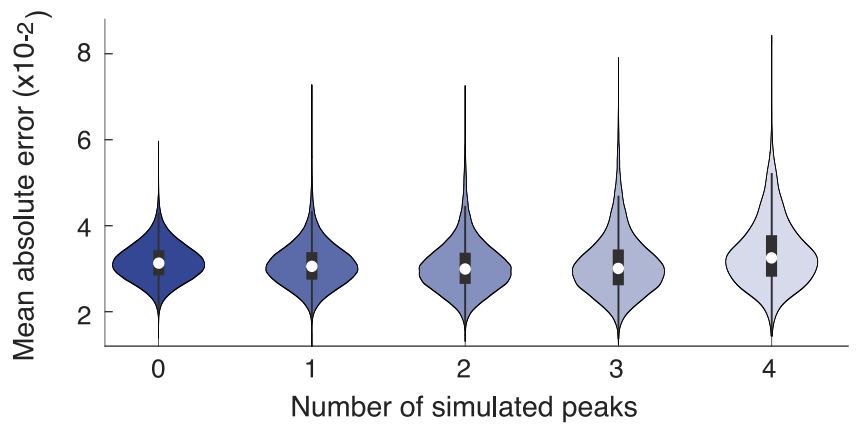

218 Figure 3: SPRiNT raw performance (Simulation Challenge II).

219 (A) SPRiNT parameterized spectrogram for a representative simulated time series with time-

220 varying aperiodic (offset, exponent) and transient periodic (centre frequency, amplitude, standard 
221 deviation) components. The red arrow indicates a cross-sectional view of the spectrogram at 14

222 s. (B) Absolute error in SPRiNT parameter estimates across all simulations $(n=10,000)$. (C)

223 Detection probability of spectral peaks (i.e., rhythmic components) depending on simulated

224 centre frequency and amplitude (light blue: 3-8 Hz theta; yellow: 8-13 Hz alpha; orange: 13-18

$225 \mathrm{~Hz}$ beta). (D) Number of fitted vs. simulated rhythmic components (spectral peaks), across all

226 simulations and time points. The underestimation of the number of estimated spectral peaks is

227 related to centre frequency: 3-8 Hz simulated peaks (blue) account for proportionally fewer of

228 recovered peaks between 3-18 Hz (blue, yellow, orange) than from the other two tertiles.

229 (Samples sizes by number of simulated peaks: 0 peaks $=798,753,1$ peak $=256,599,2$ peaks $=$

$23078,698,3$ peaks $=14,790,4$ peaks $=1,160)(\mathrm{E})$ Model fit error is not affected by number of

231 simulated peaks. Violin plots show the full sample distributions (white circle: median, grey box:

232 first and third quartiles; whiskers: range).

233 Figure supplement 1. SPRiNT raw performance (without outlier peak removal).

Aperiodic and periodic fluctuations in resting-state EEG dynamics with eyes-closed, eyes-

\section{6 open behaviour (empirical data).}

237 We applied SPRiNT to resting-state EEG data from the openly available LEMON dataset 238 (Babayan et al., 2019). Participants $(n=178)$ were instructed to open and close their eyes 239 (alternating every $60 \mathrm{~s}$ ). We used Brainstorm (Tadel et al., 2011) to preprocess EEG time series 240 from electrode $\mathrm{Oz}$ and obtain time-frequency decompositions with Morlet wavelets (with default 241 parameters, see Methods). We ran SPRiNT on the same preprocessed EEG time series. 
bioRxiv preprint doi: https://doi.org/10.1101/2022.01.21.477243; this version posted February 9, 2022. The copyright holder for this preprint (which was not certified by peer review) is the author/funder, who has granted bioRxiv a license to display the preprint in perpetuity. It is made available under aCC-BY-NC-ND 4.0 International license.

244 eyes (Figure 4A and Figure 4 - figure supplement 1). These changes in the alpha band were also

245 visible in the wavelet spectrogram. We found the wavelet estimate of alpha band signal power to

246 be associated with the experimental condition $(\beta=-1.99, \mathrm{SE}=0.30,95 \% \mathrm{CI}[-2.61,-1.42]$; Bayes

247 factor $\mathrm{BF}=5.00 \times 10^{-11}$; Table 1$)$. As expected, time points of reduced alpha power predicted that

248 participants had their eyes open.

249

a) time-frequency decompositions: LEMON dataset
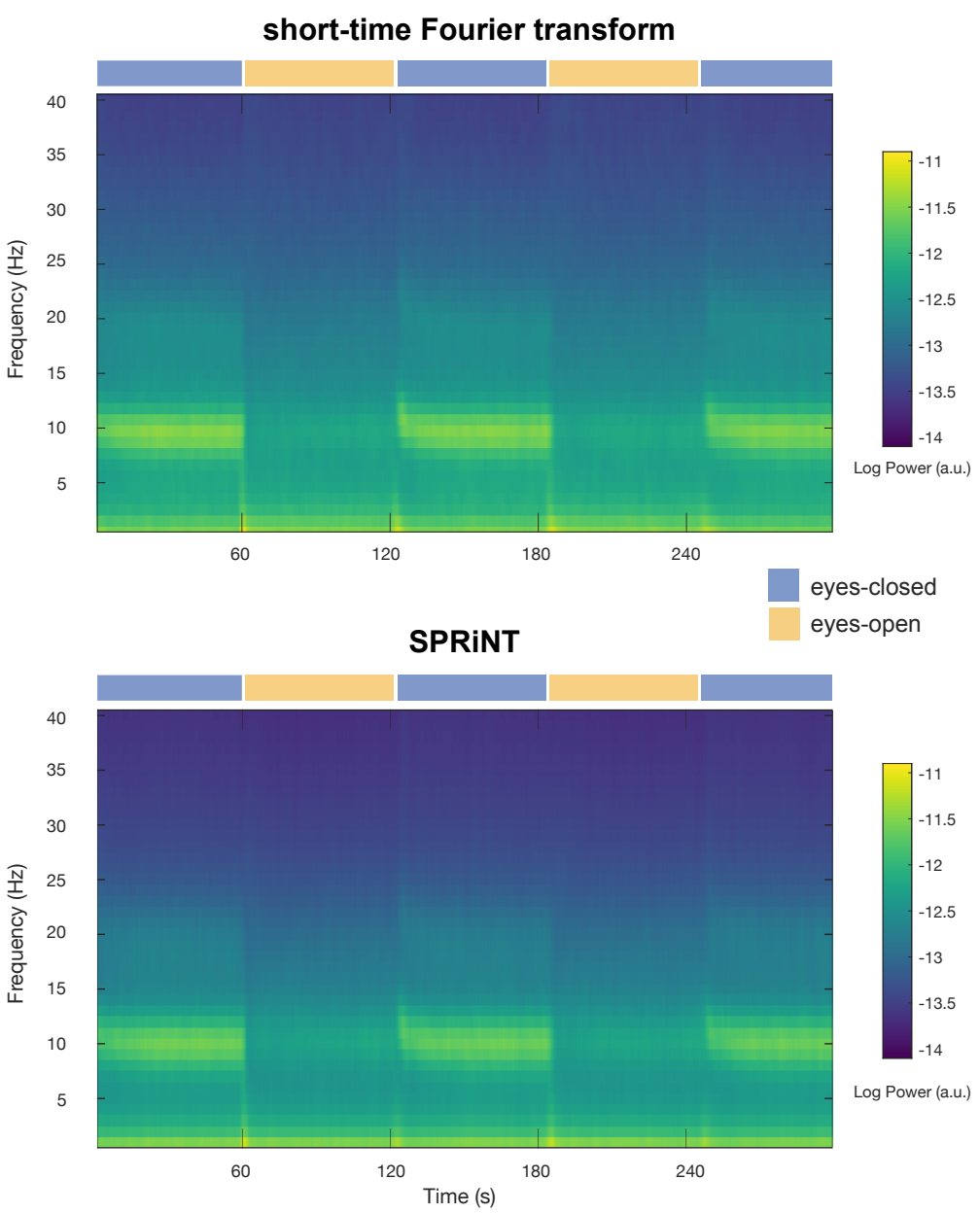

eyes-closed eyes-open

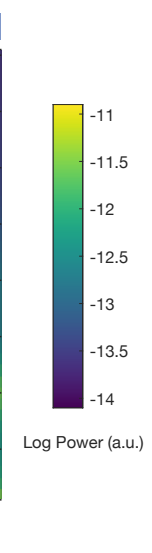

b) age effects on eyes-closed EEG

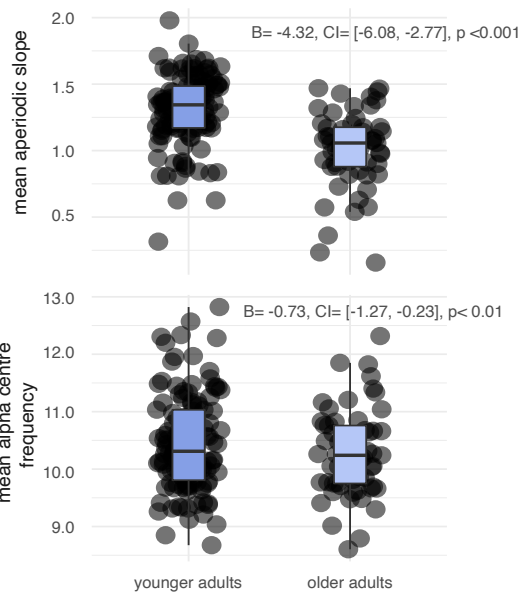

c) age effects on eyes-open EEG

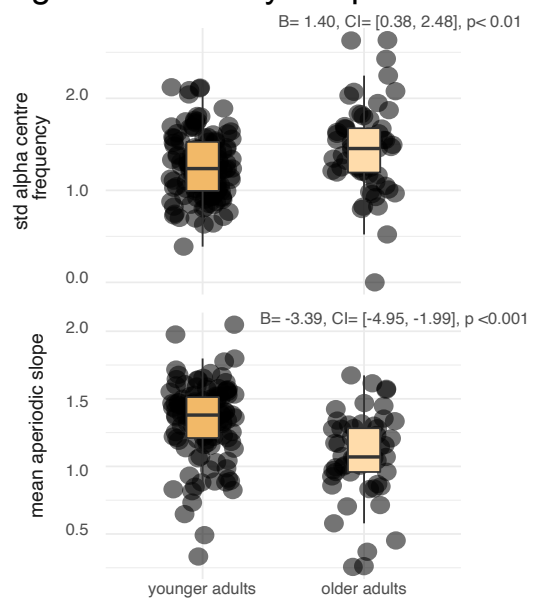

251 Figure 4: SPRiNT parameterization of resting-state electroencephalography

252 (A) We generated spectrograms across all participants $(n=178)$ over 5 min of continuous eyes-

253 closed-eyes-open recordings from electrode Oz, using both short-time Fourier transforms (top) 
and SPRiNT (bottom). (B) Logistic regression models indicated that mean aperiodic exponent and mean alpha centre frequency parameters in eyes-closed resting-state data are predictive of age group. (C) Logistic regression models indicated that mean aperiodic exponent and variability

257 (standard deviation) of alpha centre frequency parameters in eyes-closed resting-state data are 258 predictive of age group (boxplot line: median; boxplot limits: first and third quartiles; whiskers:

259 range). Sample sizes: younger adults (age: 20-40 years): 121; older adults (age: 55-80 years): 57.

260 Figure supplement 1. SPRiNT model parameters in resting-state EEG.

261 Figure supplement 2. Bayes factor analysis for predicting experimental condition (eyes-open

262 vs. eyes-closed).

263 Figure supplement 3. Bayes factor analysis for eyes-open resting-state brain activity.

264 Figure supplement 4. Bayes factor analysis for eyes-closed resting-state brain activity.

We ran a similar logistic regression model with SPRiNT parameter estimates as fixed 267 effects (mean and standard deviation of alpha centre frequency, alpha power, and the aperiodic 268 exponent across time). We found a significant effect of mean alpha power $(\beta=-6.31, \mathrm{SE}=0.90$, $26995 \%$ CI $[-8.20,-4.64])$, standard deviation of alpha power $(\beta=5.57, \mathrm{SE}=1.94,95 \% \mathrm{CI}[1.86$, $2709.51])$, and mean aperiodic exponent $(\beta=2.44, \mathrm{SE}=0.52,95 \% \mathrm{CI}[1.45,3.50])$ as predictors of 271 eyes-open vs. eyes-closed behaviour (Table 2). According to this model, lower alpha power, larger 272 aperiodic exponents, and stronger fluctuations of alpha-band activity over time are signatures of 273 the eyes-open resting-state. A Bayes factor analysis confirmed the evidence of effects from mean 274 alpha power $\left(\mathrm{BF}=1.14 \times 10^{-13}\right)$ and mean aperiodic exponent $\left(\mathrm{BF}=2.49 \times 10^{-4}\right)$, and indicated 275 anecdotal evidence against the variability of alpha power $(\mathrm{BF}=1.18$; Figure 4 - figure supplement 276 2). In summary, both wavelet and SPRiNT analyses confirmed alpha power as a 
neurophysiological marker of eyes-closed vs. eyes-open behavior. Yet SPRiNT's spectrogram parametrization was uniquely able to reveal time-varying changes of aperiodic components related to eyes-closed vs. eyes-open behavior. with participants' age group (i.e., younger $[n=121]$ vs older $[n=57]$ adults). Extant literature reports slower alpha rhythms and smaller aperiodic exponents in healthy aging (Donoghue et al., 2020). We performed a logistic regression based on SPRiNT parameter estimates of the mean and standard deviation of alpha centre frequency, alpha power, and aperiodic exponent as fixed effects in the eyes-open condition. We found significant effects of mean aperiodic exponent $(\beta=-3.39$, $\mathrm{SE}=0.75,95 \% \mathrm{CI}[-4.95,-1.99])$ and standard deviation of alpha centre frequency $(\beta=1.40, \mathrm{SE}$

$290=0.53,95 \% \mathrm{CI}[0.38,2.48]$; Table 3). We therefore found using SPRiNT that the EEG spectrogram

291 of older participants was flatter and expressed stronger time-varying fluctuations of alpha-peak 292 centre frequency. A Bayes factor analysis showed strong evidence for the effect of the aperiodic 293 exponent $\left(\mathrm{BF}=2.38 \times 10^{-5}\right)$ and for the variability of the alpha peak centre frequency $(\mathrm{BF}=0.13$;

294 Figure 4 - figure supplement 3).

We replicated the same analysis with the data in the eyes-closed condition. We found with

296 SPRiNT that mean aperiodic exponent $(\beta=-4.32, \mathrm{SE}=0.84,95 \% \mathrm{CI}[-6.08,-2.77])$ and mean

297 alpha centre frequency $(\beta=-0.73, \mathrm{SE}=0.26,95 \%$ CI $[-1.27,-0.23])$ were predictors of 298 participants' age group, with older participants again showing a flatter spectrum and a slower alpha 299 peak (lower centre frequency; Table 4). A Bayes factor analysis provided strong evidence for the 
effect of mean aperiodic exponent $\left(\mathrm{BF}=1.21 \times 10^{-7}\right)$ and strong evidence for the effect of mean

301 alpha centre frequency $(\mathrm{BF}=0.07$; Figure $4-$ figure supplement 4$)$.

Using the wavelet spectrogram alternative, we found that mean alpha power was the only

303 discriminant feature for age group in the eyes-closed condition $(\beta=-1.14, \mathrm{SE}=0.38,95 \% \mathrm{CI}[-$

304 1.92, -0.41]; Table 5). Contrasting with SPRiNT's present findings, the effect of age was confined

305 to alpha power using wavelet spectrograms. Because wavelet spectrograms are not readily

306 decomposed into aperiodic and periodic components, their findings could be alternatively

307 interpreted as caused by age-related effects on aperiodic exponent or alpha-peak centre frequency

308 (as discussed by Scally et al., 2018). There was no wavelet-based predictor of age group in the

309 eyes-open condition (Table 6).

a) subject EC012
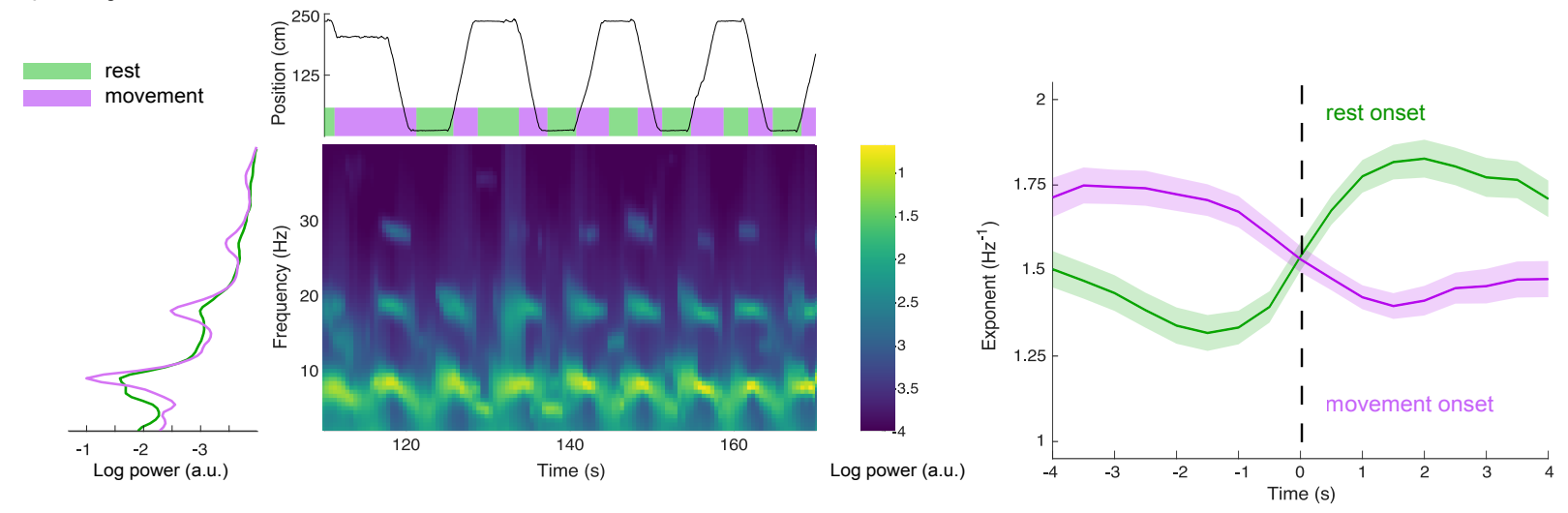

b) subject EC013
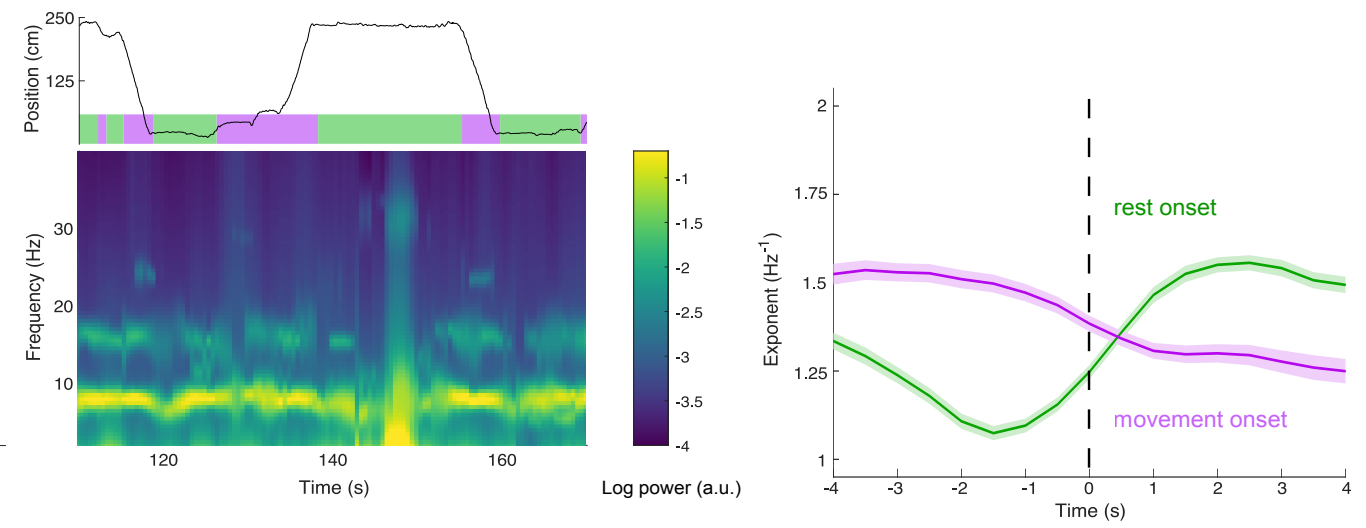


\section{Figure 5: SPRiNT captures aperiodic dynamics related to locomotion}

313 (A) We generated power spectra collapsed across rest (green) and movement (purple) periods for

314 subject EC012, observing broad increases in spectral power in frequencies below $20 \mathrm{~Hz}$ during

315 rest relative to movement. A sample SPRiNT model spectrogram is provided, with position time

316 series located above (green: rest; purple: movement). We observed gradual shifts around

317 locomotor transitions of aperiodic exponent (right), with increasing exponents at the onset of rest

318 and decreasing exponents at movement onset (solid line: mean; shaded region: 95\% CI). (B)

319 Same data as (A), but for subject EC013. Sample sizes: EC012 rest onset (green): 67 epochs;

320 EC012 movement onset (purple): 89 epochs; EC013 rest onset: 323 epochs; EC013 movement

321 onset: 263 epochs.

322 Figure supplement 1. Examples of sawtooth rhythms from two representative electrodes in 323 entorhinal cortex layer 3 from both subjects.

324 Figure supplement 2. Empirical distributions of SPRiNT aperiodic exponent and offset

325 parameters.

328 Transient changes in aperiodic brain activity associated with rodent locomotor behaviour

329 (empirical data).

330 We used intracranial data from two Long-Evans rats recorded in layer 3 of entorhinal cortex while

331 they moved freely along a linear track (Mizuseki et al., 2009; https://crcns.org). Rats travelled

332 alternatively to either end of the track to receive a water reward, resulting in behaviours of

333 recurring bouts of running and resting. Power spectral density estimates revealed substantial power

334 increases below $20 \mathrm{~Hz}$ during rest with respect to movement (Figure 5; Samiee \& Baillet, 2017). 
335 We therefore tested for the possible expression of two alternating modes of aperiodic neural

336 activity associated with each behaviour. SPRiNT parameterization found in the two subjects that

337 resting bouts were associated with larger aperiodic exponents and more positive offsets than during

338 movement bouts (Figure 5 - figure supplement 2). We ran SPRiNT parameterizations over eight-

339 second epochs proximal to transitions between movement and rest, and observed dynamic shifts

340 between aperiodic modes associated with behavioural changes (Figure 5). We found that the

341 periodic features of the recordings were non-sinusoidal and therefore should not be parametrized

342 with the methods discussed herein (Donoghue et al., 2021; Figure 5 - figure supplement 1).

\section{Discussion}

346 We introduce SPRiNT as a new method to parameterize dynamic fluctuations in the spectral

347 contents of neural time series. SPRiNT extends recent practical tools that determine aperiodic and

348 periodic parameters from static power spectrum density estimates of neural signals to their

349 spectrograms. Aperiodic spectral components may confound the detection and interpretation of 350 narrow-band power changes as periodic, oscillatory signal elements. Given the scientific 351 prominence of measures of neural oscillations in (causal) relation to behaviour (e.g., Albouy et al., 352 2017) and clinical syndromes (e.g., Ostlund et al., 2021), it is essential that their characterization 353 in time and frequency be contrasted with that of the underlying aperiodic background activity at 354 the natural time scale of behaviour and perception. 
357 Recent empirical studies show that the spectral distribution of neural signal power with frequency

358 can be decomposed into low-dimensional aperiodic and periodic components (Donoghue et al.,

359 2020), and that these latter are physiologically (Gao et al., 2019), clinically (Molina et al., 2020;

360 Van Heumen et al., 2021), and behaviourally (Ouyang et al., 2020; Waschke et al., 2021)

361 meaningful. neural spectrograms. The method combines the simplicity of the specparam spectral

364 decomposition approach with the computational efficiency of short-time Fourier transforms across

365 sliding windows. The present results demonstrate its technical concept and indicate that SPRiNT

366 unveils meaningful additional information from the data, beyond established tools such as wavelet

367 time-frequency decompositions.

Using realistic simulations of neural time series, we demonstrate the strengths and current

369 limitations of SPRiNT. We show that SPRiNT decompositions provide a comprehensive account

370 of the neural spectrogram (Figure 2A), tracking the dynamics of periodic and aperiodic signal

371 components across time (Figure $2 \mathrm{~B}$ and Figure 2 - figure supplement 1 ). We note that the

372 algorithm performs optimally when the data features narrow-band oscillatory components that can

373 be characterized as spectral peaks (Figure 3C). The algorithm performs best when the data contains

374 two or fewer salient oscillatory components concurrently (Figure 3D). We found that these current

375 limitations are inherent to specparam, which is challenged by the dissociation of spectral peaks

376 from background aperiodic activity at the lower edge of the power spectrum (Donoghue et al., 377 2020)

378 Practical mitigation techniques have been proposed to account for the presence of 379 background aperiodic activity when estimating narrow-band signal power changes. For instance, 
baseline normalization is a common approach used to isolate event-related signals and prepare spectrograms for comparisons across individuals (Cohen, 2014). However, the resulting relative measures of event-related power increases or decreases do not explicitly account for the fact that

383 behaviour or stimulus presentations may induce rapid changes in aperiodic activity. Therefore,

384 baseline normalization followed by narrow-band analysis of power changes is not immune to 385 interpretation ambiguities when aperiodic background activity also changes dynamically. Further, 386 the definition of a reference baseline can be inadequate for some study designs, as exemplified 387 herein with the LEMON dataset.

\section{SPRiNT decomposition of EEG data tracks and predicts behaviour \& demographics}

390 We found in the LEMON dataset that measuring narrow band power changes without accounting

391 for concurrent variations of the aperiodic signal background challenges the interpretation of effects

392 manifested in the spectrogram (Scally et al., 2018). SPRiNT enables this distinction, showing that

393 both periodic and aperiodic changes in neural activity are associated with age and behaviour. We

394 found strong evidence for decreases in alpha-peak power and increases in aperiodic exponent

395 during eyes-open resting-state behaviour (compared to eyes-closed; Figure 4A). However, it

396 remains unclear whether these effects are independent or related; a recent analysis of the same

397 dataset showed that the amplitude of alpha oscillations around a non-zero mean voltage influence

398 baseline cortical excitability (Studenova et al., 2021) — an effect observable in part through

399 variations of the aperiodic exponent (Gao et al., 2017). We also observed both slower alpha-peak

400 centre frequencies and smaller aperiodic exponents in the older age group (Figure 4B), in

401 agreement with previous literature on healthy aging (Cellier et al., 2021; Donoghue et al., 2020;

402 Hill et al., 2022; Ostlund et al., 2022; Schaworonkow \& Voytek, 2021). 
We also emphasize how the variability of spectral parameters may relate to demographic

404 features, as shown with SPRiNT's prediction of participants' age from the variability of alpha-

405 peak centre frequency over the duration of the recording (Figure 4C). Previous studies explored

406 similar effects of within-subject variability of alpha peak centre frequency (Haegens et al., 2014)

407 and their clinical relevance (Larsson \& Kostov 2005). These findings augment the recent evidence

408 that neural spectral features are robust signatures proper to an individual (da Silva Castanheira et

409 al., 2021) and open the possibility that their temporal variability be neurophysiologically

410 significant.

411 We also report time-resolved fluctuations in aperiodic activity related to behaviour in

412 freely moving rats (Figure 5). SPRiNT aperiodic parameters highlight larger spectral exponents

413 in rats during rest than during movement. Time-resolved aperiodic parameters can also be

414 tracked with SPRiNT as subjects transition from periods of movement to rest and vice-versa. The

415 smaller aperiodic exponents observed during movement may be indicative of periods of general

416 cortical disinhibition (Gao et al., 2017). Previous work on the same data has associated how

417 locomotor behavior is associated with changes in amplitude and centre frequency of entorhinal

418 theta rhythms (Mizuseki et al., 2009; Samiee \& Baillet, 2017). Strong theta activity may

419 challenge the estimation of aperiodic parameters (Gao et al., 2017). The nature and function of

420 the changes in aperiodic exponent with behaviour detected with SPRiNT demonstrated in the

421 present data remain to be determined, but these observations support the notion that aperiodic

422 background neural activity changes in relation to a variety of contexts and subject types

423 (Donoghue et al., 2020; Gao et al., 2017; Molina et al., 2020; Ostlund et al., 2021; Pathania et al.,

424 2021; Waschke et al., 2021; van Heumen et al., 2021). 


\section{Future directions}

427 We used the short-time Fourier transform as the underlying time-frequency decomposition

428 technique for SPRiNT. A major asset of STFT is computational efficiency, but with sliding time

429 windows of fixed duration, the method demonstrates the trade-off between temporal specificity

430 and frequency resolution (Cohen, 2014). Combining specparam with STFT yields rapid extraction

431 of spectral parameters from time-frequency data. In principle, spectral parameterization should be

432 capable of supplementing any time-frequency decomposition technique, such as wavelet

433 transforms (Pietrelli et al., 2021), though at the expense of significantly greater computational cost.

434 However, we have shown with the wavelet-specparam method herein that parameterizing wavelet

435 transforms does not accurately recover aperiodic signal components. Further, at the levels of

436 temporal smoothing necessary to reduce parameter estimation error to similar levels as SPRiNT's

437 (4-s Gaussian kernel; Figure 2), the high temporal sampling of wavelet spectral parameterization

438 becomes redundant. The advantages to such an approach over parameterized STFTs (i.e., SPRiNT)

439 are unclear, because parameter estimates are accurate even with relatively low time and frequency

440 resolutions $(0.5 \mathrm{~s}$ and $1 \mathrm{~Hz}$ as shown in Figures $2 \& 3)$. Another alternative to STFT would be the

441 superlet approach (Moca et al., 2021) designed to preserve a fixed resolution across time (number

442 of cycles) and frequency. Combining superlets with specparam is to be explored, although reduced

443 computational cost remains a practical benefit of STFT.

SPRiNT has some limitations similar to specparam (Donoghue et al., 2020). For example,

445 the determination of periodic component parameters (number of peaks, peak amplitude) may be

446 prone to overfitting the number of peaks in the data. Here, we propose a pragmatic approach to

447 this issue with a postprocessing step that consists in pruning the outcome of original SPRiNT

448 parameters from unlikely, transient periodic components (Figure 1 - figure supplement 1). 
449 Identifying the most parsimonious number of oscillatory peak components in the data (via model

450 selection e.g., via Bayesian Information Criteria; Schwarz, 1978) may be an approach worth

451 exploring in that respect.

452 In conclusion, the SPRiNT algorithm enables the parameterization of the neural

453 spectrogram. We validated the time tracking of periodic and aperiodic spectral features with a

454 large sample of ground-truth synthetic time series, and empirical data from the human resting-

455 state, and rodent intracranial electrophysiological recordings. We showed that SPRiNT provides

456 estimates of dynamic fluctuations of aperiodic and periodic neural activity that are related to

457 meaningful demographic or behavioural outcomes. We anticipate that SPRiNT and future related

458 developments will augment the neuroscience toolkit and enable new advances in the

459 characterization of complex neural dynamics. 


\section{Methods}

463 SPRiNT runs on individual time series and returns a parameterized time-frequency representation

464 of the spectrogram. The algorithm first derives short-time fast Fourier transforms (FFTs) over time

465 windows that slide on the time series. Second, the modulus of FFT coefficients is averaged over $n$

466 consecutive time windows to produce smoothed power spectral density (PSD) estimates at each

467 time bin. Third, each of the resulting PSDs are parameterized into periodic and aperiodic

468 components, using the specparam algorithm. A fourth optional step consists of the removal of

469 outlier periodic components from the raw SPRiNT spectrograms. We developed SPRiNT as a

470 plug-in library that interoperates with Brainstorm (Tadel et al., 2011).

\section{Parameterization of short-time FFT}

473 Short-time Fourier transforms are computed iteratively on sliding time windows (default window

474 length $=1 \mathrm{~s}$; tapered by a Hann window) using MATLAB's Fast Fourier Transform (FFT; R2020a;

475 Natick, MA). Each window overlaps with its nearest neighbours (default overlap $=50 \%$ ). The 476 modulus of Fourier coefficients of the running time window is then averaged locally with those

477 from preceding and following time windows, with the number of time windows included in the 478 average, $n$, determined by the user (default is $n=5$; Figure $1 \mathrm{~A}$ ). The resulting periodogram is then 479 parameterized with specparam. The resulting spectrogram is time-binned based on time points 480 located at the centre of each sliding time window.

482 Tracking periodic and aperiodic components across time

483 We used the MATLAB implementation of specparam in Brainstorm (Tadel et al., 2011), adapted 484 from the original Python code (Version 1.0.0) by Donoghue et al. (2020). The aperiodic component 
485 of the power spectrum is typically represented using two parameters (exponent and offset); an

486 additional knee parameter is added when a bend is present in the aperiodic component (Donoghue

487 et al., 2020; Gao et al., 2020). Periodic components are parameterized as peaks emerging from the

488 aperiodic component using Gaussian functions controlled with three parameters (mean [centre

489 frequency], amplitude, and standard deviation).

490 For algorithmic speed optimization purposes, in each iteration of specparam across time, the

491 optimization of the aperiodic exponent is initialized from its specparam estimate from the

492 preceding time bin. All other parameter estimates are initialized using the same data-driven

493 approaches as specparam (Donoghue et al., 2020).

\section{Pruning of periodic component outliers}

496 We derived a procedure to remove occasional peaks of periodic activity from parameterized

497 spectrograms and emphasize expressions of biologically plausible oscillatory components across

498 successive time bins. This procedure removes peaks with fewer than a user-defined number of

499 similar peaks (by centre frequency; default $=3$ peaks within $2.5 \mathrm{~Hz}$ ) within nearby time bins

500 (default $=6$ bins). This draws from observations in synthetic data that non-simulated peaks are

501 parameterized in isolation (few similar peaks in neighbouring time bins; Figure 1 - figure

502 supplement 1). Aperiodic parameters are refit at time bins where peaks have been removed, and 503 models are subsequently updated to reflect changes in parameters. This post-processing procedure 504 is applied on all SPRiNT outputs shown but remains optional (albeit recommended). 
507 We simulated neural time series using in-house code based on the NeuroDSP toolbox (Cole et al.,

508 2019) with MATLAB (R2020a; Natick, MA). The time series combined aperiodic with periodic

509 components (Donoghue et al., 2020). Each simulated 60-s time segment consisted of white noise

510 sampled at $200 \mathrm{~Hz}$ generated with MATLAB's coloured noise generator (R2020a; Natick, MA).

511 The time series was then Fourier-transformed (frequency resolution $=0.017 \mathrm{~Hz}$ ) and convolved

512 with a composite spectrogram of simulated aperiodic and periodic dynamics (temporal resolution

$513=0.005 \mathrm{~s}$ ). The final simulated time series was generated as the linear combination of cosines of

514 each sampled frequency (with random initial phases), with amplitudes across time corresponding

515 to the expected power from the spectrogram.

\section{Simulations of transient and chirping peak components}

518 The aperiodic exponent was initialized to $1.5 \mathrm{~Hz}^{-1}$ and increased to $2.0 \mathrm{~Hz}^{-1}$, offset was initialized 519 to -2.56 a.u. and increased to -1.41 a.u.; both linearly increasing between the $24 \mathrm{~s}$ and $36 \mathrm{~s}$ time 520 stamps of the time series. Periodic activity in the alpha band (centre frequency $=8 \mathrm{~Hz}$, amplitude $521=1.2$ a.u., standard deviation $=1.2 \mathrm{~Hz}$ ) was generated between time stamps $8 \mathrm{~s}$ and $40 \mathrm{~s}$, as well as 522 between 41-46 s and 47-52 s. Periodic activity in the beta band (centre frequency $=18 \mathrm{~Hz}$, 523 amplitude $=0.9$ a.u., standard deviation $=1.4 \mathrm{~Hz}$ ) was generated between $15-25 \mathrm{~s}$ and down524 chirped linearly from $18-15 \mathrm{~Hz}$ between $18-22$ s. Peak amplitude was calculated as the relative 525 height above the aperiodic component at every sampled frequency and time point. All amplitudes 526 of periodic activity were tapered by a Tukey kernel (cosine fraction $=0.4)$. Aperiodic and periodic 527 parameters (and their dynamics) were combined to form a spectrogram of simulated activity. 
All simulations $(n=10,000)$ were unique as each was generated from a unique white-noise time series seed, and the cosine waves to simulate periodic components were each assigned a random initial phase. with $50 \%$ overlap (frequency range: $1-40 \mathrm{~Hz}$ ). Settings for specparam were set as: peak width

533 limits: [0.5 6]; maximum number of peaks: 3; minimum peak amplitude: 0.6 a.u.; peak threshold:

5342.0 standard deviations; proximity threshold: 2 standard deviations; aperiodic mode: fixed.

535 Settings for peak post-processing were set as: number of neighbouring peaks: 3; centre frequency:

$5362.5 \mathrm{~Hz}$; time bin: 6 bins (= $3 \mathrm{~s})$. Periodic alpha activity was identified using the highest amplitude

537 peak parameterized in each time bin between $5.5-10.5 \mathrm{~Hz}$, while periodic beta activity was 538 identified using the highest amplitude peak in each time bin between 13.5-20.5 Hz. specparam (Donoghue et al., 2020; MATLAB version). Wavelet transforms were computed with Brainstorm (Tadel et al., 2011; 1-40 Hz, in $1 \mathrm{~Hz}$ steps) using default settings (central frequency =

$5423 \mathrm{~Hz}, \mathrm{FWHM}=1 \mathrm{~s}$ ). Before parameterizing wavelet transforms, we applied a $4 \mathrm{~s}$ temporal 543 smoothing filter (Gaussian kernel, standard deviation $=1 \mathrm{~s}$; time range: $3.5-56.5 \mathrm{~s}$, in $0.005 \mathrm{~s}$ steps)

544 to increase signal-to-noise ratio (results prior to this step are shown for the first 1,000 simulations

545 in Supplemental Materials). Settings for specparam were set as: peak width limits: [0.5 6];

546 maximum number of peaks: 3; minimum peak amplitude: 0.6 a.u.; peak threshold: 2.0 standard

547 deviations; proximity threshold: 2 standard deviations; aperiodic mode: fixed. Periodic alpha

548 activity was identified using the highest amplitude peak parameterized in each time bin between

$549 \quad 5.5-10.5 \mathrm{~Hz}$. Periodic beta activity was identified using the highest amplitude peak in each time

550 bin between $13.5-20.5 \mathrm{~Hz}$. 
Model fit error was calculated as the mean absolute error (MAE) between expected and

553 modelled spectral power by each component across simulations and times. Algorithmic

554 performance was assessed by calculating MAE in parameter estimates across simulations and

555 times relative to expected parameters. Peak-fitting probability in the alpha $(5.5-10.5 \mathrm{~Hz})$ and beta

$556(13.5-20.5 \mathrm{~Hz})$ bands were calculated for each time bin as the fraction of simulations with at least

557 one oscillatory peak recovered in the frequency band of interest.

\section{Generic time series simulations}

560 For each time series generation, we sampled the parameter values of their a/rhythmic components

561 uniformly from realistic ranges. Aperiodic exponents were initialized between $0.8-2.2 \mathrm{~Hz}^{-1}$.

562 Aperiodic offsets were initialized between -8.1 and -1.5 a.u. Within the 12-36 s time segment into

563 the simulation (onset randomized), the aperiodic exponent and offset underwent a linear shift of

564 magnitude in the ranges -0.5 to $0.5 \mathrm{~Hz}^{-1}$ and -1 to 1 a.u. (sampled continuously, chosen randomly),

565 respectively. The duration of the linear shift was randomly selected for each simulated time series

566 between 6 and $24 \mathrm{~s}$. Between 0-4 oscillatory (rhythmic) components were added to each trial with

567 parameters randomly sampled within the following ranges: centre frequency: 3-35 Hz; amplitude:

568 0.6-1.6 a.u.; standard deviation: 1-2 Hz. The onset (5-40 s) and duration (3-20 s) of each of the

569 rhythmic components were also randomized across components and across trials, with the

570 constraint that they would not overlap both in time and frequency; they were allowed to overlap

571 in either dimension. If a rhythmic component overlapped temporally with another one, its centre

572 frequency was set at least 2.5 peak standard deviations from the other temporally overlapping 
573 rhythmic component(s). The magnitude of each periodic component was tapered by a Tukey kernel

$574 \quad($ cosine fraction $=0.4)$.

575 Each simulation was analyzed with SPRiNT using 5x1s STFT windows with 50\% overlap

576 (frequency range: 1-40 Hz). Settings for specparam were: peak width limits: [0.5 6]; maximum

577 number of peaks: 6; minimum peak amplitude: 0.6 a.u.; peak threshold: 2.0 standard deviations;

578 proximity threshold: 2 standard deviations; aperiodic mode: fixed. Settings for peak post-

579 processing were: number of neighbouring peaks: 3 ; centre frequency: $2.5 \mathrm{~Hz}$; time bin: 6 bins (=

5803 s). The spectrogram outcome of SPRiNT was analyzed to identify rhythmic components as

581 correct (i.e., present in ground truth signal) or incorrect components. Rhythmic SPRiNT

582 components were labelled as correct if their centre frequency was within 2.5 peak standard

583 deviations from any of the ground truth rhythmic components. In the event of multiple SPRiNT

584 rhythmic components meeting these conditions, we selected the one with the largest amplitude

585 peak (marking the other as incorrect).

Errors on parameter estimates were assessed via MAE measures with respect to their

587 ground truth values. The peak-fitting probability for each simulated rhythmic component was

588 derived as the fraction of correct peaks recovered when one was expected. Model fit error was

589 calculated for each time bin as the MAE between empirical and SPRiNT spectral power. We

590 used a linear regression model (fitlm MATLAB function; 2020a; Natick, MA) to predict model

591 fit errors at each time bin, using number of simulated peaks as a predictor.

$$
M A E=\text { intercept }+B * \text { number of simulated peaks }
$$




\section{Study 2: Resting-state electrophysiology data}

596 We used open-access resting-state electroencephalography (EEG) and demographics data 597 collected for 212 participants from the Leipzig Study on Mind-Body-Emotion Interactions

598 (LEMON; Babayan et al., 2019). Data from the original study was collected in accordance with

599 the Declaration of Helsinki and the study protocol was approved by the ethics committee at the

600 medical faculty of the University of Leipzig. Participants were asked to alternate every 60 seconds

601 between eyes-open and eyes-closed resting-state for 16 minutes. Continuous EEG activity (2500-

$602 \mathrm{~Hz}$ sampling rate) was recorded from $61 \mathrm{Ag} / \mathrm{AgCl}$ active electrodes placed in accordance with the

603 10-10 system. An electrode below the right eye recorded eye-blinks (ActiCap System, Brain

604 Products). Impedance of all electrodes was maintained below $5 \mathrm{k} \Omega$. EEG recordings were

605 referenced to $\mathrm{FCz}$ during data collection (Babayan et al., 2019), and re-referenced to an average

606 reference during preprocessing.

607 Preprocessing was performed using Brainstorm (Tadel et al., 2011). Recordings were 608 resampled to $250 \mathrm{~Hz}$ before being high-pass filtered at $0.1 \mathrm{~Hz}$ using a Kaiser window. Eyeblink 609 EEG artifacts were detected and removed using SSP projectors. Data was visually inspected for 610 bad channels and artifacts exceeding $200 \mu \mathrm{V} .20$ participants were excluded for not following task

611 instructions, 2 for failed recordings, 1 for data missing event markers, and 11 were excluded for 612 EEG data of poor quality ( $>8$ bad sensors). The results herein are from the remaining 178 613 participants (average number of bad sensors $=3$ ). We extracted the first 5 minutes of consecutive 614 quality data, beginning with the eyes-closed condition, from electrode Oz for each participant. 
617 Each recording block was analyzed with SPRiNT using 5x1s sliding time windows with 50\%

618 overlap (frequency range: $1-40 \mathrm{~Hz}$ ). We ran SPRiNT using the Brainstorm implementation with

619 the following settings: peak width limits: [1.5 6]; maximum number of peaks: 3; minimum peak

620 amplitude: 0.5 a.u.; peak threshold: 2.0 standard deviations; proximity threshold: 2.5 standard

621 deviations; aperiodic mode: fixed. Peak post-processing was run on SPRiNT outputs (number of

622 neighbouring peaks 3; centre frequency: $2.5 \mathrm{~Hz}$; time bin: 6 bins (= $3 \mathrm{~s})$. Alpha peaks were defined

623 as all oscillatory peaks parameterized between 6-14 Hz. To capture variability in alpha peak centre

624 frequency across time, mean and standard deviations of alpha peak centre frequency distributions

625 were computed across both eyes -open and -closed conditions and by age group (defined below).

626 We also computed spectrograms from Morlet wavelet time-frequency decompositions (1-

$62740 \mathrm{~Hz}$, in $1 \mathrm{~Hz}$ steps) also using Brainstorm (with default parameters; central frequency $=1 \mathrm{~Hz}$,

$628 \quad$ FWHM = 3 s; Tadel et al., 2011).

Contrast between eyes-open and eyes-closed conditions

631 All regression analyses were performed in R (V 3.6.3; R Core Team, 2020). We ran a logistic

632 regression model whereby we predicted the condition (i.e., eyes-open vs eyes-closed) from the

633 mean and standard deviation of the following SPRiNT parameters: alpha centre frequency, alpha

634 power, and aperiodic exponent. All model predictors were entered as fixed effects. Significance of

635 each beta coefficient was tested against zero (i.e., $B_{n}=0$ ). We quantified the evidence for each

636 predictor in our models with a Bayes factor analysis where we systematically removed one of the

637 predictors and computed the Bayes factor using the BayesFactor library (Morey \& Rouder, 2018).

638 We compared the most complex model (i.e., the full model) against all models formulated by

639 removing a single predictor. Evidence in favor of the full model (i.e., $\mathrm{BF}<1$ ) indicated that a given 
predictor improved model fit, whereas evidence for the model without the predictor (i.e., BF > 1) showed limited improvement in terms of model fit.

We also fitted a logistic regression model to predict experimental condition (i.e., eyes open \& -closed; dummy coded) from mean alpha-band power $(6-14 \mathrm{~Hz})$ entered as a fixed effect.

644 Alpha-band power was computed as the mean log-power between 6-14 Hz for each condition 645 extracted from the Morlet wavelets spectrograms. Significance of each beta coefficient was tested against zero (i.e., $B_{n}=0$ ).

\section{Predicting age from resting-state activity}

649 Participants were assigned to two groups based on their biological age: younger adults (age: 20-

65040 years, $n=121$ ) and older adults (age: 55-80 years, $n=57$ ). Modelled alpha peaks and aperiodic

651 parameters were collapsed across time to generate condition-specific distributions of model

652 parameters per participant. We used these distributions to examine the mean and standard

653 deviation of alpha centre frequency, alpha power, and aperiodic exponent. We fitted two logistic

654 regression models using the glm function in R (R Core Team, 2020) for the eyes-open and eyes-

655 closed conditions:

$$
\begin{aligned}
\text { age }=\text { intercept } & +\mathrm{B} 1 * \text { mean alpha center frequency }+\mathrm{B} 2 \\
& * \text { standard deviation alpha center frequency }+\mathrm{B} 3 * \text { mean alpha power }+\mathrm{B} 4 \\
& * \text { standard deviation alpha power }+\mathrm{B} 5 * \text { mean aperiodic slope }+\mathrm{B} 6 \\
& * \text { standard deviation aperiodic slope }
\end{aligned}
$$

All predictors were entered as fixed effects. Significance of each beta coefficient was tested a Bayes factor analysis.

\section{Study 3: Intracranial rodent data}


667 Local field potential (LFP) recordings and animal behaviour, originally published by Mizuseki et

668 al. (2009), were collected from 2 Long-Evans rats (data retrieved from https://crcns.org). Animals

669 were implanted with eight-shank multi-site silicon probes (200 $\mu \mathrm{m}$ inter-shank distance) spanning

670 multiple layers of dorsocaudal medial entorhinal cortex (entorhinal cortex, dentate gyrus,

671 hippocampus). Neurophysiological signals were recorded while animals traversed to alternating

672 ends of an elevated linear track $(250 \times 7 \mathrm{~cm})$ for $30 \mu \mathrm{L}$ water reward (animals were water deprived

673 for $24 \mathrm{hrs}$ prior to task). All surgical and behavioural procedures in the original study were

674 approved by the Institutional Animal Care and Use Committee of Rutgers University. Recordings

675 were acquired continuously at $20 \mathrm{kHz}$ (RC Electronics) and bandpass-filtered (1 Hz-5 kHz) before

676 being down-sampled to $1250 \mathrm{~Hz}$. In two rats (EC012, EC013), nine recording blocks of activity in

677 entorhinal cortex layer 3 (EC3) were selected for further analysis (16 electrodes in EC012, 8

678 electrodes in EC013). Data from one electrode in EC012 was removed for having poor quality.

679 Animal head position was extracted from video recordings $(39.06 \mathrm{~Hz})$ of two head-mounted LEDs

680 and temporally interpolated to align with SPRiNT parameters across time (piecewise cubic

681 Hermite interpolative polynomial; pchip in MATLAB; 2020a; Natick, MA).

\section{Spectrogram analysis}

684 Each recording block was analyzed with SPRiNT using 5x2 s sliding time windows with 75\%

685 overlap (frequency range: 2-40 Hz). Settings for specparam were set as: peak width limits: [1.5 5];

686 maximum number of peaks: 3; minimum peak amplitude: 0.5 a.u.; peak threshold: 2.0 standard

687 deviations; proximity threshold: 2.0 standard deviations; aperiodic mode: fixed. Settings for peak

688 post-processing were set as: number of neighbouring peaks: 3 ; centre frequency bounds: $2.5 \mathrm{~Hz}$; 
689 time bin bounds: 6 bins (= 3 s). Aperiodic parameters were averaged across electrodes and aligned

690 with behavioural data.

692 Tracking aperiodic dynamics during movement transitions

693 Time bins were categorized based on whether animals were resting at either end of the track or

694 moving toward opposite ends of the track ('rest' or 'movement', respectively) using animal

695 position (and speed). Rest-to-movement and movement-to-rest transitions were defined as at least

696 four consecutive seconds of rest followed by four consecutive seconds of run $(t=0$ representing

697 the onset of movement $)$, or vice-versa $(t=0$ representing the onset of rest), respectively.

\section{Software and code availability}

701 The SPRiNT algorithm and all code needed to produce the figures shown are available from 702 GitHub (https://github.com/lucwilson/SPRiNT). The SPRiNT algorithm is also available from the 703 Brainstorm distribution (Tadel et al., 2011).

\section{Data availability}

706 The simulated data are openly available on OSF (https://osf.io/c3gn4/). Resting-state EEG data 707 was obtained from the open repository LEMON (Babayan et al., 2019; 708 https://openneuro.org/datasets/ds000221/versions/00002). Intracranial rodent data (study HC3) is 709 openly available from Mizuseki et al. (2009; data retrieved from https://crcns.org). SPRiNT710 processed data is available upon request from the corresponding author. 


\section{Author contributions}

713 All authors conceptualized the study, L.E.W. and J.D.S.C. preformed the analyses, S.B. provided 714 guidance with data interpretation, all authors contributed to the writing and editing of the 715 manuscript.

716

\section{Competing interests}

718 The authors declare no competing financial interest.

\section{Acknowledgements}

721 L.E.W. acknowledges the support of an NSERC Undergraduate Student Research Award.

722 J.D.S.C. acknowledges the support of the Alexander Graham-Bell Doctoral NSERC fellowship.

723 S.B. is grateful for the support received from the NIH (R01 EB026299), a Discovery Grant from

724 the Natural Science and Engineering Research Council of Canada (436355-13), the CIHR Canada

725 Research Chair in Neural Dynamics of Brain Systems, the Brain Canada Foundation with support

726 from Health Canada, and the Innovative Ideas program from the Canada First Research Excellence

727 Fund, awarded to McGill University for the Healthy Brains for Healthy Lives initiative. This

728 research was undertaken thanks in part to funding from the Canada First Research Excellence

729 Fund, awarded to McGill University for the Healthy Brains for Healthy Lives initiative.

\section{References}

732 Albouy, P., Weiss, A., Baillet, S., \& Zatorre, R. J. (2017). Selective Entrainment of Theta

733 Oscillations in the Dorsal Stream Causally Enhances Auditory Working Memory 
Alhourani, A., Wozny, T. A., Krishnaswamy, D., Pathak, S., Walls, S. A., Ghuman, A. S., ... Niranjan, A. (2016). Magnetoencephalography-based identification of functional connectivity network disruption following mild traumatic brain injury. J Neurophysiol, 116(4), 1840-1847. doi:10.1152/jn.00513.2016

Babayan, A., Erbey, M., Kumral, D., Reinelt, J. D., Reiter, A. M. F., Röbbig, J., . . Villringer, A. (2019). A mind-brain-body dataset of MRI, EEG, cognition, emotion, and peripheral physiology in young and old adults. Scientific Data, 6(1), 180308.

Bruns, A. (2004). Fourier-, Hilbert- and wavelet-based signal analysis: are they really different approaches? Journal of Neuroscience Methods, 137(2), 321-332. doi:10.1016/j.jneumeth.2004.03.002

Buzsáki, G. (2006). Rhythms of the brain. Oxford: Oxford University Press.

747 Buzsáki, G., \& Watson, B. O. (2012). Brain rhythms and neural syntax: implications for efficient coding of cognitive content and neuropsychiatric disease. Dialogues in clinical neuroscience, 14(4), 345-367. doi: 10.31887/DCNS.2012.14.4/gbuzsaki

750 Cellier, D., Riddle, J., Petersen, I., \& Hwang, K. (2021). The development of theta and alpha neural oscillations from ages 3 to 24 years. Developmental Cognitive Neuroscience, 50, 100969.

753 Chini, M., Pfeffer, T., \& Hanganu-Opatz, I. L. (2021). Developmental increase of inhibition drives decorrelation of neural activity. bioRxiv, 2021.2007.2006.451299. doi:10.1101/2021.07.06.451299

Cohen, M. X. (2014). Analyzing neural time series data: Theory and practice. Boston:

$$
\text { MIT Press. }
$$


758 Cole, S., Donoghue, T., Gao, R., \& Voytek, B. (2019). NeuroDSP: A package for neural

759

760

761

762

763

764

765

766

767

768

769

770

771

772

773

774

775

776

777

778

digital signal processing. Journal of Open Source Software, 4(36), 1272.

doi:10.21105/joss.01272

da Silva Castanheira, J., Orozco Perez, H. D., Misic, B., \& Baillet, S. (2021). Brief segments of neurophysiological activity enable individual differentiation. Nature Communications, 12(1), 5713. doi:10.1038/s41467-021-25895-8

Donoghue, T., Haller, M., Peterson, E. J., Varma, P., Sebastian, P., Gao, R., . . Knight, R. T. (2020). Parameterizing neural power spectra into periodic and aperiodic components. Nature Neuroscience, 23(12), 1655-1665. doi:10.1038/s41593-020-00744-x

Donoghue, T., Schaworonkow, N., \& Voytek, B. (2021). Methodological considerations for studying neural oscillations. Eur J Neurosci. doi:10.1111/ejn.15361

Gao, R., Peterson, E. J., \& Voytek, B. (2017). Inferring synaptic excitation/inhibition balance from field potentials. NeuroImage, 158, 70-78. doi:10.1016/j.neuroimage.2017.06.078

Haegens, S., Cousijn, H., Wallis, G., Harrison, P. J., \& Nobre, A. C. (2014). Inter- and intraindividual variability in alpha peak frequency. NeuroImage, 92, 46-55. doi:10.1016/j.neuroimage.2014.01.049

He, B. J. (2014). Scale-free brain activity: Past, present, and future. Trends in Cognitive Sciences, 18(9), 480-487. doi:10.1016/j.tics.2014.04.003

Hill, A. T., Clark, G. M., Bigelow, F. J., Lum, J. A. G., \& Enticott, P. G. (2022). Periodic and aperiodic neural activity displays age-dependent changes across early-to-middle childhood. Developmental Cognitive Neuroscience, 54, 101076. doi:10.1016/j.den.2022.101076 
Kucewicz, M. T., Cimbalnik, J., Matsumoto, J. Y., Brinkmann, B. H., Bower, M. R., Vasoli, V., ... Worrell, G. A. (2014). High frequency oscillations are associated with cognitive processing in human recognition memory. Brain, 137(8), 2231-2244. doi:10.1093/brain/awu149

Larsson, P. G., \& Kostov, H. (2005). Lower frequency variability in the alpha activity in EEG among patients with epilepsy. Clinical Neurophysiology, 116(11), 2701-2706. doi:10.1016/j.clinph.2005.07.019

Mizuseki, K., Sirota, A., Pastalkova, E., \& Buzsáki, G. (2009). Theta Oscillations Provide Temporal Windows for Local Circuit Computation in the Entorhinal-Hippocampal Loop. Neuron, 64(2), 267-280. doi:10.1016/j.neuron.2009.08.037

Moca, V. V., Bârzan, H., Nagy-Dăbâcan, A., \& Mureșan, R. C. (2021). Time-frequency superresolution with superlets. Nature Communications, 12(1), 337. doi:10.1038/s41467-02020539-9

Molina, J. L., Voytek, B., Thomas, M. L., Joshi, Y. B., Bhakta, S. G., Talledo, J. A., .. . Light, G. A. (2020). Memantine Effects on Electroencephalographic Measures of Putative Excitatory/Inhibitory Balance in Schizophrenia. Biol Psychiatry Cogn Neurosci Neuroimaging, 5(6), 562-568. doi:10.1016/j.bpsc.2020.02.004

Morey, R. D., \& Rouder, J. N. (2018). BayesFactor: Computation of Bayes factors for common designs. R package version 0.9.12-4.2. Retrieved from https://CRAN.R-project.org/package=BayesFactor Ostlund, B. D., Alperin, B. R., Drew, T., \& Karalunas, S. L. (2021). Behavioral and cognitive correlates of the aperiodic (1/f-like) exponent of the EEG power spectrum in adolescents with and without ADHD. Developmental Cognitive Neuroscience, 48, 100931. doi:10.1016/j.den.2021.100931 
Ostlund, B., Donoghue, T., Anaya, B., Gunther, K. E., Karalunas, S. L., Voytek, B., \& Pérez-Edgar, K. E. (2022). Spectral parameterization for studying neurodevelopment: How and why. Developmental Cognitive Neuroscience, 54, 101073. doi:10.1016/j.dcn.2022.101073 brain activities reveals their differential associations with cognitive processing speed. NeuroImage, 205, 116304. doi:10.1016/j.neuroimage.2019.116304

Pathania, A., Schreiber, M., Miller, M. W., Euler, M. J., \& Lohse, K. R. (2021). Exploring the reliability and sensitivity of the EEG power spectrum as a biomarker. International

Pietrelli, M., Samaha, J., \& Postle, B. R. (2021). Spectral distribution dynamics across different attentional priority states. bioRxiv, 2021.2012.2002.470964. doi:10.1101/2021.12.02.470964

R Core Team. (2020). R: A language and environment for statistical computing. R Foundation

814 Samaha, J., Iemi, L., Haegens, S., \& Busch, N. A. (2020). Spontaneous Brain Oscillations and Perceptual Decision-Making. Trends in Cognitive Sciences, 24(8), 639-653. doi:10.1016/j.tics.2020.05.004

817 Samiee, S., \& Baillet, S. (2017). Time-resolved phase-amplitude coupling in neural oscillations. NeuroImage, 159, 270-279. doi:10.1016/j.neuroimage.2017.07.051

819 Scally, B., Burke, M. R., Bunce, D., \& Delvenne, J.-F. (2018). Resting-state EEG power and 820 connectivity are associated with alpha peak frequency slowing in healthy aging. 
822 Schaworonkow, N., \& Voytek, B. (2021). Longitudinal changes in aperiodic and periodic

823

824

825

826

827

828

829

830

831

832

833

834

835

836

837

838

839

840

841

842

activity in electrophysiological recordings in the first seven months of life.

Developmental Cognitive Neuroscience, 47, 100895. doi:10.1016/j.dcn.2020.100895

Schwarz, G. (1978). Estimating the Dimension of a Model. The Annals of Statistics, 6(2), 461464. doi:10.1214/aos/1176344136

Studenova, A. A., Villringer, A., \& Nikulin, V. V. (2021). Baseline shift in neuronal oscillations and its implications for the interpretation of evoked activity obtained with EEG/MEG. bioRxiv, 2021.2012.2001.470793. doi:10.1101/2021.12.01.470793

Tadel, F., Baillet, S., Mosher, J. C., Pantazis, D., \& Leahy, R. M. (2011). Brainstorm: A UserFriendly Application for MEG/EEG Analysis. Computational Intelligence and Neuroscience, 2011, 879716. doi:10.1155/2011/879716

van Heumen, S., Moreau, J. T., Simard-Tremblay, E., Albrecht, S., Dudley, R. W., \& Baillet, S. (2021). Case Report: Aperiodic Fluctuations of Neural Activity in the Ictal MEG of a Child With DrugResistant Fronto-Temporal Epilepsy. Frontiers in Human Neuroscience, 15(101). doi:10.3389/fnhum.2021.646426

Voytek, B., \& Knight, R. T. (2015). Dynamic Network Communication as a Unifying Neural Basis for Cognition, Development, Aging, and Disease. Biological Psychiatry, 77(12), 1089-1097. doi:10.1016/j.biopsych.2015.04.016

Voytek, B., Kramer, M. A., Case, J., Lepage, K. Q., Tempesta, Z. R., Knight, R. T., \& Gazzaley, A. (2015). Age-Related Changes in 1/f Neural Electrophysiological Noise. The Journal of Neuroscience, 35(38), 13257. doi:10.1523/JNEUROSCI.2332-14.2015 
843 Waschke, L., Donoghue, T., Fiedler, L., Smith, S., Garrett, D. D., Voytek, B., \& Obleser, J. (2021).

844 Modality-specific tracking of attention and sensory statistics in the human electrophysiological

845 spectral exponent. eLife, 10, e70068. doi:10.7554/eLife.70068

846 Wen, H., \& Liu, Z. (2016). Separating fractal and oscillatory components in the power spectrum of

847 neurophysiological signal. Brain Topography, 29(1), 13-26. doi:10.1007/s 10548-015-0448-0 


\section{Supplemental Material}

\section{Detection and removal of spectrogram outlier components}

3 A known issue of specparam is the fitting of spurious, outlier spectral peaks (Donoghue et al., 4 2020). In SPRiNT, these peaks often appear as transient blips of periodic activity in the 5 spectrogram. We propose a post-processing option in SPRiNT to detect and remove transient

6 periodic activity (Figure 1 - figure supplement 1). In short, the procedure searches for clusters of

7 spectral peaks over a user-defined maximum time period (see Methods). Once an outlier peak is

8 detected at a given time bin and removed from the model, aperiodic parameters are refit with

9 specparam to account for the variance previously attributed to the spurious peak and models are

10 subsequently updated to reflect changes in parameters.

a) original SPRiNT spectrogram

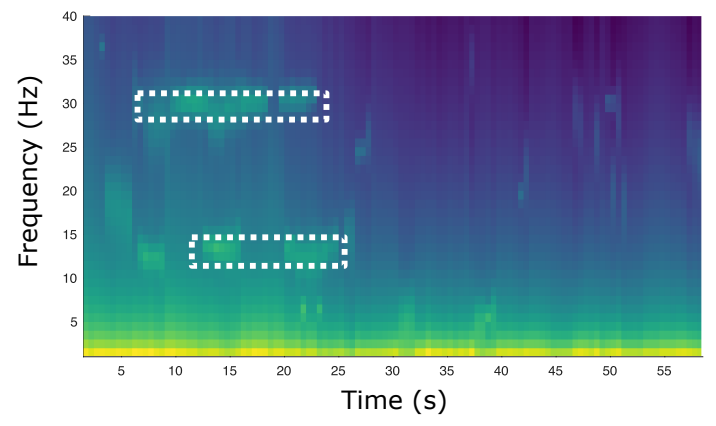

Distribution of SPRiNT peak centre frequencies

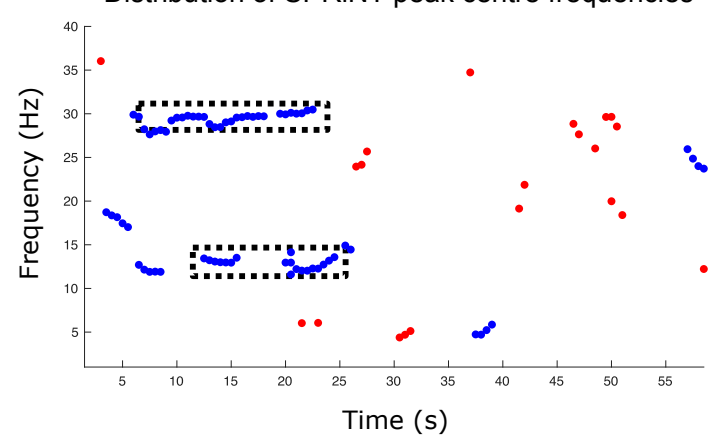

b) SPRiNT spectrogram with outliers removed

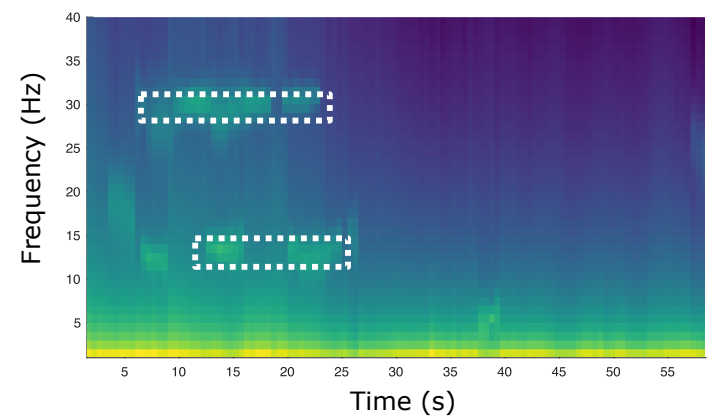

SPRiNT peak centre frequencies following outlier removal

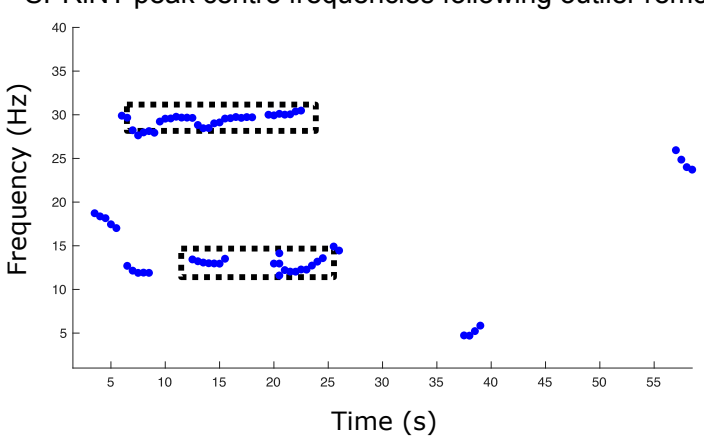

11

12 Figure 1 - figure supplement 1. Overview of the outlier peak removal process. 
13 (A) Original SPRiNT spectrogram (top) and time-frequency distribution of peak centre frequencies

14 (bottom). In this example, the detected peaks (red and blue dots) are sequentially removed if they

15 are not part of a cluster of contiguous peaks within a time-frequency region of $3 \mathrm{~s}$ and $2.5 \mathrm{~Hz}$ (red

16 dots; size of minimum cluster is adjustable by user). (B) Resulting SPRiNT spectrogram (top) and

17 peak centre frequencies (bottom) after outlier removal and the update of aperiodic parameters.

18 Dashed rectangular areas show time-frequency regions where rhythmic activity was simulated in

19 the synthesized signal.

\section{Methods benchmarking (synthetic data Challenge I)}

22 Below we present the results of SPRiNT from synthetic data Challenge I without outlier peak

23 removal. We show, similar to the results presented in the main text, that SPRiNT modelled

24 aperiodic and periodic spectrograms with low error (aperiodic model mean absolute error [MAE]

$25=0.06$; peak model MAE $=0.03$ ). For the aperiodic component, the algorithm accurately recovered

26 both ground truth exponent $(\mathrm{MAE}=0.11)$ and offset $(\mathrm{MAE}=0.15)$. It also detected the

27 occurrences of alpha peaks with high sensitivity ( $99 \%$ at time bins with maximum peak amplitude)

28 and specificity ( $6 \%$ spurious detections), and with low errors on their centre frequency (MAE =

$290.33)$ and amplitude $(\mathrm{MAE}=0.20)$ parameters, but overestimated the width of the periodic peak

30 components $(\mathrm{MAE}=0.42)$. The algorithm detected and tracked the down-chirping beta periodic

31 components with high sensitivity (95\% at time bins with maximum peak amplitude) but lower

32 specificity (95\%) than with outlier peak removal (98\%). Time-collapsed errors on centre frequency

$33(\mathrm{MAE}=0.44)$ and amplitude $(\mathrm{MAE}=0.17)$ parameters were low, with a tendency to overestimate

34 the width in frequency of the periodic component (MAE $=0.48)$. Results following outlier peak

35 removal are presented and discussed in the main text. 
We also parameterized power spectral densities of each simulation using specparam to assess how the results the first simulation challenge would appear if we assumed stationary spectral content across time. The PSD was computed using $1 \mathrm{~s}$ windows with $50 \%$ overlap. Settings for specparam were set as: frequency range: 1-40 Hz, in $1 \mathrm{~Hz}$ steps; peak width limits: [1.5 6];

41 deviations; proximity threshold: 2.0 standard deviations; aperiodic mode: fixed.

42 The average recovered aperiodic exponent was $1.94 \mathrm{~Hz}^{-1}$ (actual $=1.5$ to $2 \mathrm{~Hz}^{-1}$ ) and offset was -

431.64 a.u. (actual $=-2.56$ to -1.41 a.u.). The only peak recovered ( $99 \%$ sensitivity) was the alpha

44 peak, with an average center frequency of $8.09 \mathrm{~Hz}$ (actual $=8 \mathrm{~Hz}$ ), amplitude of 0.79 a.u. (actual

$45 \max =1.2 \mathrm{~Hz})$, and standard deviation of $1.21 \mathrm{~Hz}($ actual $=1.2 \mathrm{~Hz})$; no beta peak was detected

46 across all spectra.

\section{Wavelet-specparam without temporal smoothing (synthetic data Challenge I)}

48 We parameterized Morlet wavelet spectrograms (central frequency $=1 \mathrm{~Hz}, \mathrm{FWHM}=3 \mathrm{~s} ; 1-40$

$49 \mathrm{~Hz}$, in $1 \mathrm{~Hz}$ steps) of the first 1,000 simulated time series consisting of transient alpha and down-

50 chirping beta periodic activity (time range: $1.5-58.5 \mathrm{~s}$, in $0.005 \mathrm{~s}$ steps). Settings for specparam

51 were: peak width limits: [0.5 6]; maximum number of peaks: 3; minimum peak amplitude: 0.6 a.u.;

52 peak threshold: 2.0 standard deviations; proximity threshold: 2.0 standard deviations; aperiodic

53 mode: fixed. In the main text, we discuss results from temporally smoothed wavelet spectrograms

54 (see Methods). Here, we show results without temporal smoothing. 


\section{a) wavelet-specparam periodic parameter fits}

alpha peak $(5.5-10.5 \mathrm{~Hz})$
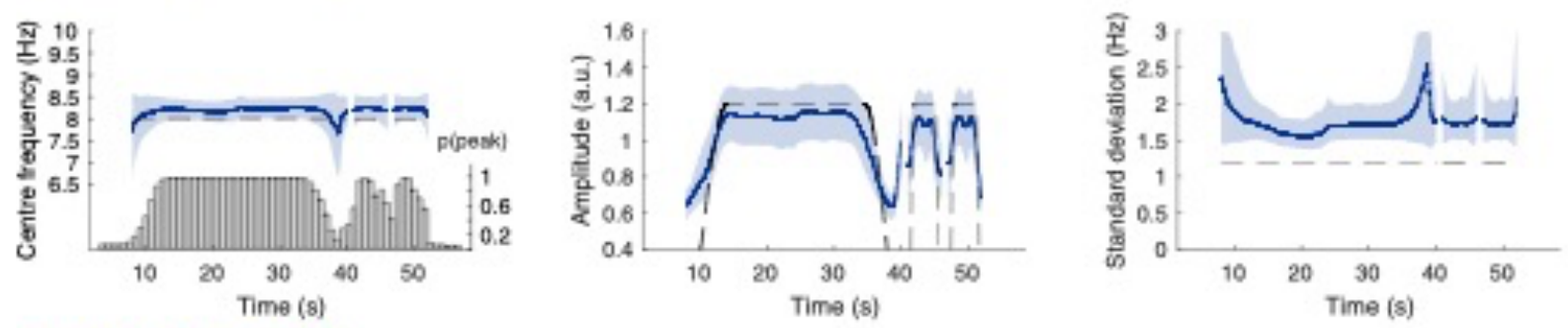

beta peak $(13.5-20.5 \mathrm{~Hz})$
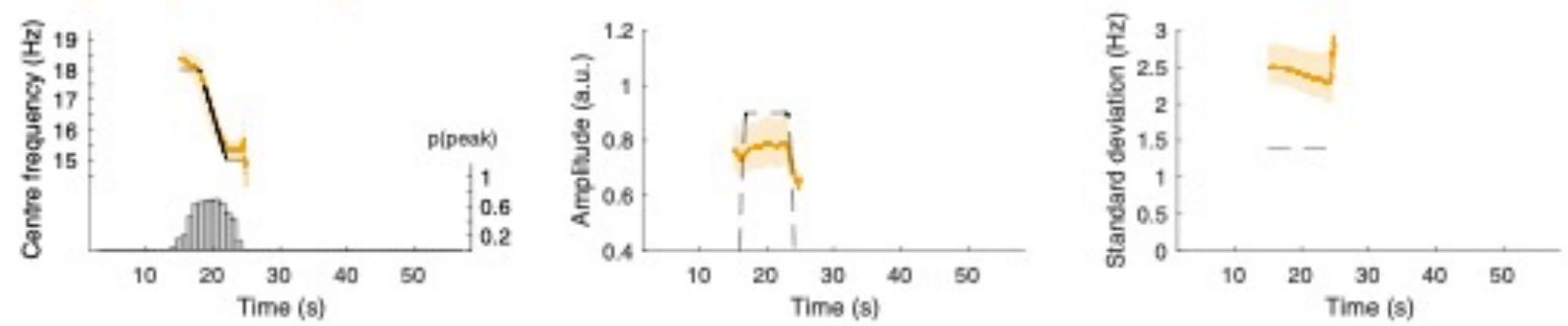

b) SPRiNT periodic parameter fits
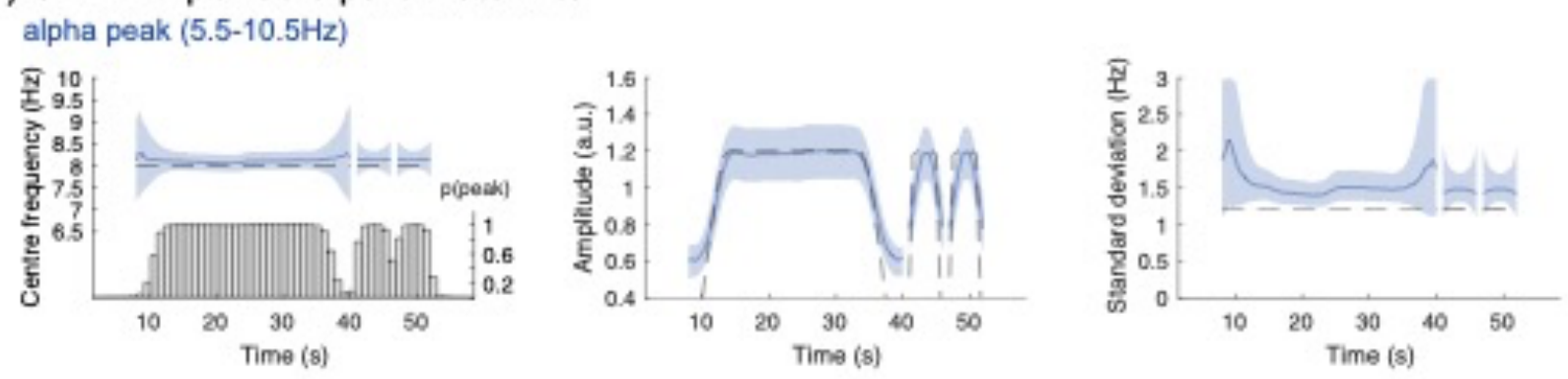

\section{beta peak $(13.5-20.5 \mathrm{~Hz})$}
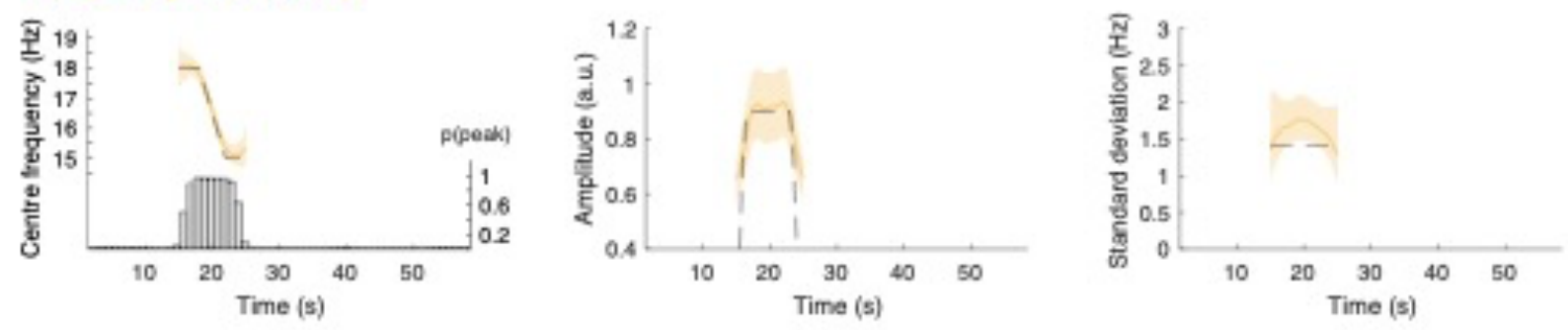

57 Figure 2 - figure supplement 1. Periodic parameter estimates across time.

58 (A) Results from the temporally smoothed wavelet-specparam approach for the alpha (top) and

59 beta (bottom) rhythmic components for each estimated parameter (from left to right: centre

60 frequency, spectral peak amplitude and standard deviation), the grey dashed line indicates ground

61 truth values over time, the plain colored line shows the median of estimates and the shaded region 
62 their first and third quartiles. The bar plots in the left panels indicate the sample probability of

63 detecting a spectral peak within respective frequency ranges at each time bin. (B) Same display

64 for the results obtained with SPRiNT. $n=10,000$ simulations.

66 Error in estimates from unsmoothed parameterized wavelet spectrograms of exponent $(\mathrm{MAE}=$

$670.41)$ and offset $(\mathrm{MAE}=0.83)$ parameters were worse than those obtained from smoothed

68 wavelet decompositions (exponent $\mathrm{MAE}=0.19$; offset $\mathrm{MAE}=0.78$ ). Alpha peaks were

69 recovered with lower sensitivity ( $84 \%$ at time bins with maximum peak amplitude) and

70 specificity ( $41 \%$ spurious detections), and with greater errors on centre frequency $(\mathrm{MAE}=0.82)$,

71 amplitude $(\mathrm{MAE}=0.53)$, and bandwidth $(\mathrm{MAE}=0.91)$. The down-chirping beta oscillation was

72 also detected with lower sensitivity (53\% at time bins with maximum peak amplitude) and

73 specificity $(74 \%)$, and with greater errors on centre frequency $(\mathrm{MAE}=1.23)$, amplitude $(\mathrm{MAE}=$

74 0.60), and bandwidth $(\mathrm{MAE}=1.10)$.

Generalization of SPRiNT across generic aperiodic and periodic fluctuations without outlier removal (synthetic data Challenge II)

79 (Figure 3 - figure supplement 1). SPRiNT recovered 70\% of the simulated periodic components,

80 with $73 \%$ specificity. Dynamic aperiodic exponents were recovered with a MAE of 0.13 , while

81 dynamic offsets were recovered with a MAE of 0.16 . Centre frequency, amplitude, and standard

82 deviation parameters were recovered with MAEs of $0.46,0.23$, and 0.49 , respectively. 
bioRxiv preprint doi: https://doi.org/10.1101/2022.01.21.477243; this version posted February 9, 2022. The copyright holder for this preprint (which was not certified by peer review) is the author/funder, who has granted bioRxiv a license to display the preprint in perpetuity. It is made available under aCC-BY-NC-ND 4.0 International license.

a) SPRiNT output example

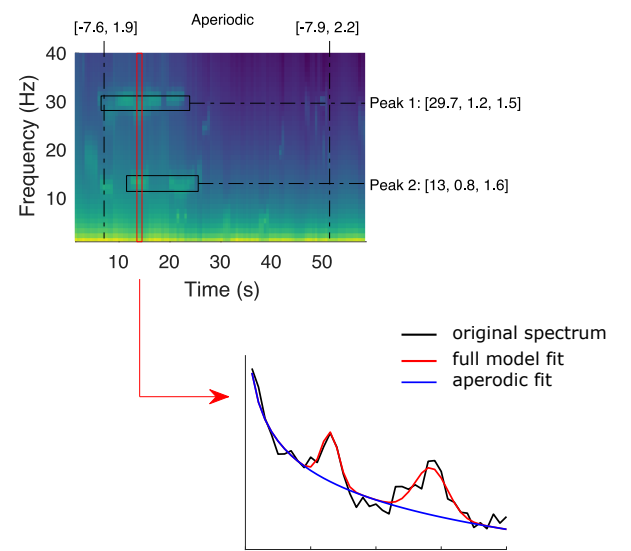

c) peak-fitting probability
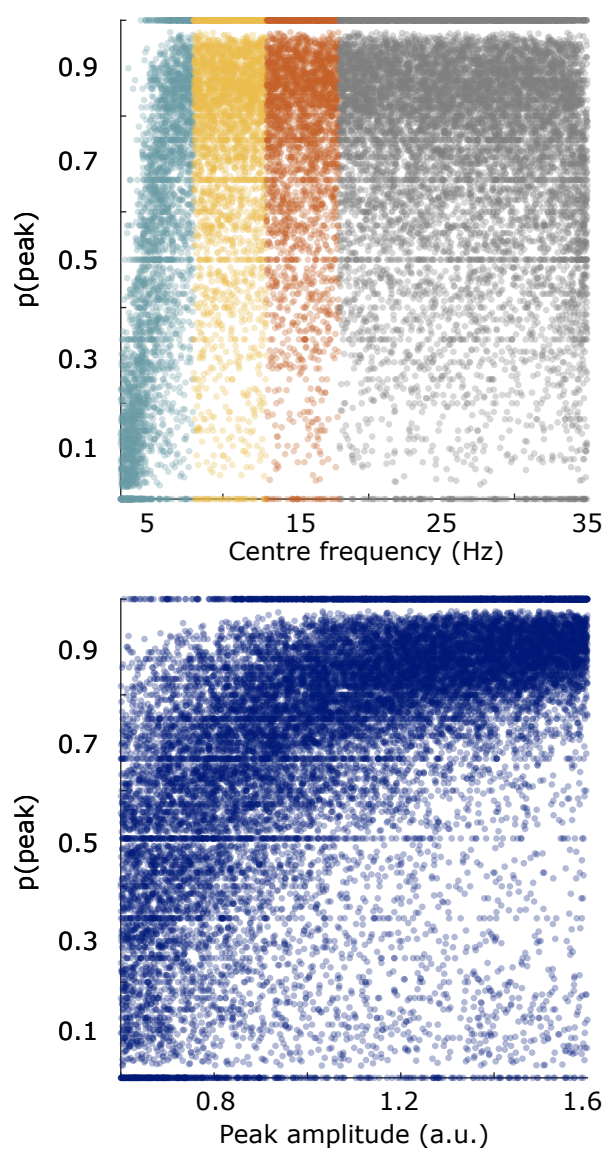

b) SPRiNT parameter errors

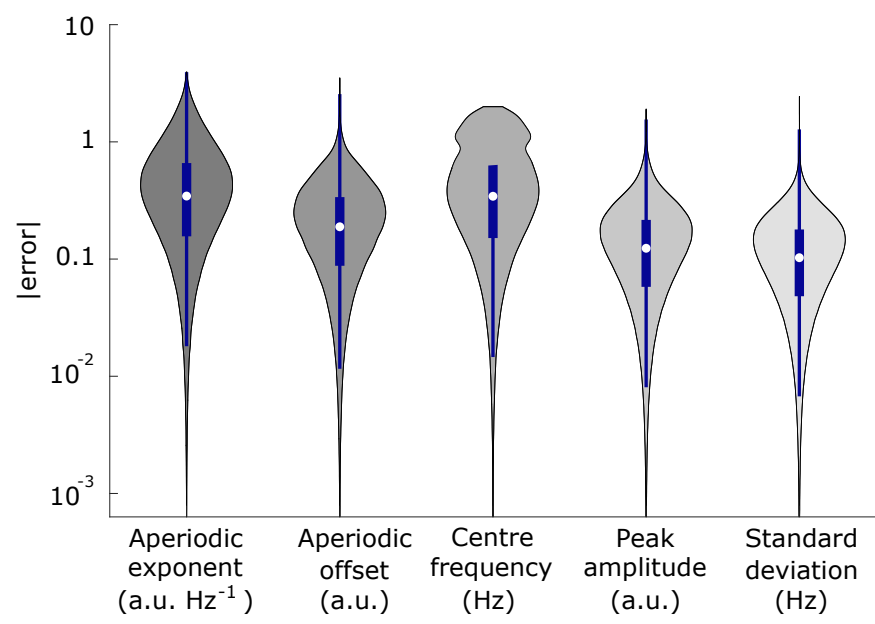

d) fitted peaks vs. actual peaks

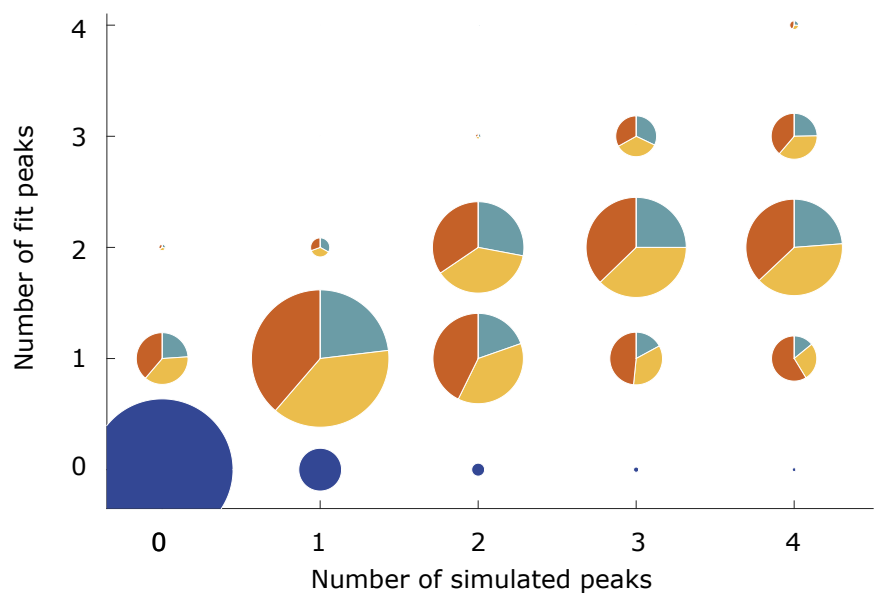

e) number of peaks does not affect model fit error

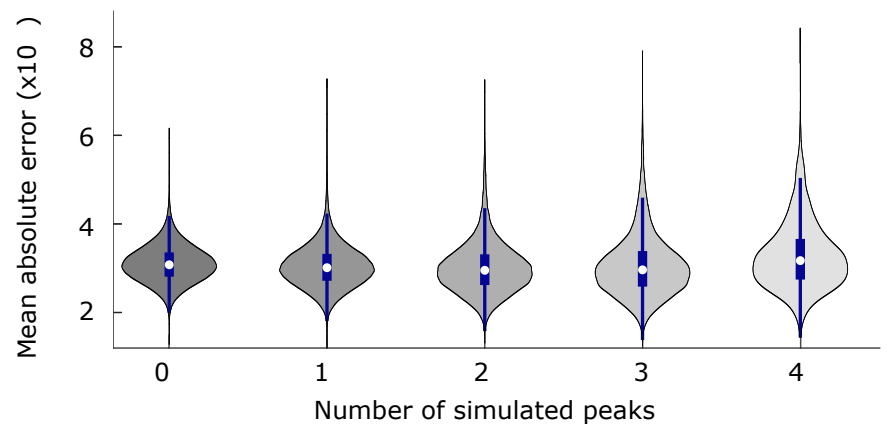

Figure 3 - figure supplement 1. SPRiNT raw performance (without outlier peak removal).

85 (A) SPRiNT parameterized spectrogram for a representative simulated time series with time-

86 varying aperiodic (offset, exponent) and transient periodic (centre frequency, amplitude, standard 
87 deviation) components. The red arrow indicates a cross-sectional view of the spectrogram at $14 \mathrm{~s}$.

88 (B) Absolute error in SPRiNT parameter estimates across all simulations $(n=10,000)$. (C)

89 Detection probability of spectral peaks (i.e., rhythmic components) depending on simulated centre

90 frequency and amplitude (light blue: 3-8 Hz theta; yellow: 8-13 Hz alpha; orange: 13-18 Hz beta).

91 (D) Number of fitted vs. simulated rhythmic components (spectral peaks), across all simulations

92 and time points. The underestimation of the number of estimated spectral peaks is related to centre

93 frequency: 3-8 Hz simulated peaks (blue) account for proportionally fewer of recovered peaks

94 between 3-18 Hz (blue, yellow, orange) than from the other two tertiles. (Samples sizes by number

95 of simulated peaks: 0 peaks $=798,753,1$ peak $=256,599,2$ peaks $=78,698,3$ peaks $=14,790,4$

96 peaks $=1,160)(\mathbf{E})$ Model fit error is not affected by number of simulated peaks. Violin plots show

97 the full sample distributions (white circle: median, blue box: first and third quartiles; whiskers:

98 range).

99

100 Aperiodic and periodic fluctuations in resting-state EEG dynamics with eyes-closed, eyes-

101 open behaviour (empirical data).

102 We ran logistics regression models to predict experimental condition with mean alpha power

103 obtained from the Morlet wavelets analysis (see Methods). Results indicated that lower alpha

104 power predicts the eyes-closed condition $(\beta=-1.99, \mathrm{SE}=0.30,95 \% \mathrm{CI}[-2.61,-1.42]$; Table 1$)$.

We fit additional regression models to predict the experimental condition (i.e., eyes-open

106 vs. eyes-closed) from SPRiNT parameters. As discussed in the main text, mean alpha power, the

107 standard deviation of alpha power, and mean aperiodic slope predicted experimental condition

108 (i.e., eyes-open vs -closed; Table 2). A Bayes factor analysis further supported these findings,

109 showcasing strong evidence for both the mean aperiodic slope and alpha power, but negative 
110 evidence (i.e., evidence for the null hypothesis) for the standard deviation of alpha power (Figure

1114 - figure supplement 3). These results, along with effects reported using wavelets (above)

112 replicate the well-documented decrease of alpha-band power in the eyes-open resting-state

113 (compared to eyes-closed). Yet, spectral parameterization highlights the merits of tracking

114 aperiodic activity, as SPRiNT captured an increase in aperiodic slope when participants closed

115 their eyes. Similarly, SPRiNT captures dynamic changes in periodic power while simultaneously

116 controlling for (and tracking) changes in the aperiodic component of the spectrogram, dissociating

117 effects driven by changes by the aperiodic alone from changes in periodic power (Donoghue et al.,

118 2020).

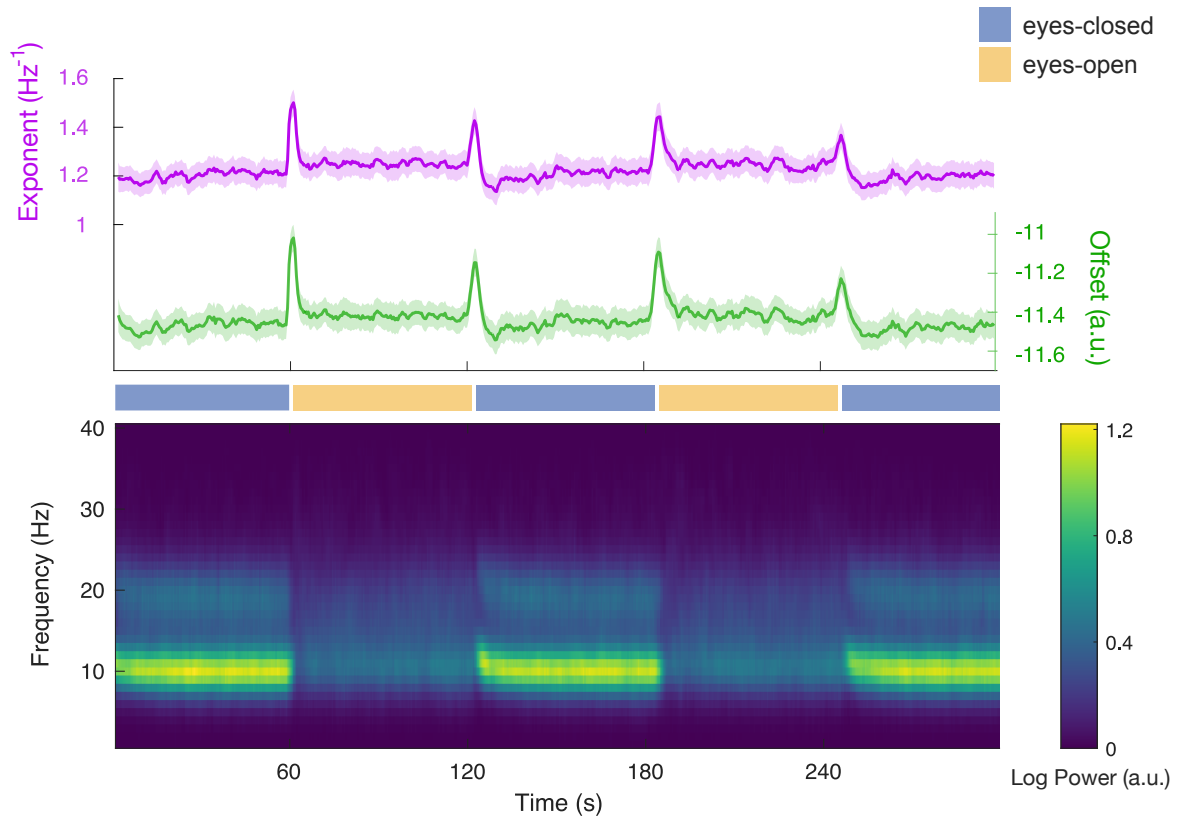

121 Figure 4 - figure supplement 1. SPRiNT model parameters in resting-state EEG. SPRiNT

122 aperiodic parameters (top panel; line: group mean $(n=178)$; shaded region: 95\% CI) and SPRiNT

123 periodic-activity averaged across participants (bottom panel). 


\begin{tabular}{lccc}
\hline \hline & \multicolumn{3}{c}{ Condition } \\
Predictors & Log-Odds & $C I$ & $p$ \\
\hline (Intercept) & -25.24 & $-33.08--17.95$ & $<\mathbf{0 . 0 0 1}$ \\
Alpha power (Morlet wavelets) & -1.99 & $-2.61--1.42$ & $<\mathbf{0 . 0 0 1}$ \\
& & & \\
\hline Observations & 356 & & \\
$\mathrm{R}^{2}$ Tjur & & &
\end{tabular}

126 Table 1. Logistic regression model parameters for predicting condition (eyes-closed vs eyes-open)

127 from the Morlet wavelet spectrograms, BF: 5.00x10 $10^{-11}$.

\section{Condition}

\begin{tabular}{lccc} 
Predictors & Log-Odds & $C I$ & $p$ \\
\hline (Intercept) & -0.20 & $-4.07-3.72$ & 0.920 \\
mean center frequency & 0.24 & $-0.04-0.53$ & 0.091 \\
std center frequency & 0.07 & $-0.82-0.97$ & 0.881 \\
mean alpha power & -6.31 & $-8.20--4.64$ & $<\mathbf{0 . 0 0 1}$ \\
std alpha power & 5.57 & $1.86-9.51$ & $\mathbf{0 . 0 0 4}$ \\
mean aperiodic slope & 2.44 & $1.45-3.50$ & $<\mathbf{0 . 0 0 1}$
\end{tabular}




\begin{tabular}{llll} 
std aperiodic slope & -3.00 & $-8.78-3.06$ & 0.315 \\
& & \\
\hline Observations & 356 & \\
$\mathrm{R}^{2}$ Tjur & 0.426
\end{tabular}

129 Table 2. Logistic regression model of SPRiNT parameters for predicting condition (eyes-closed 130 vs eyes-open).

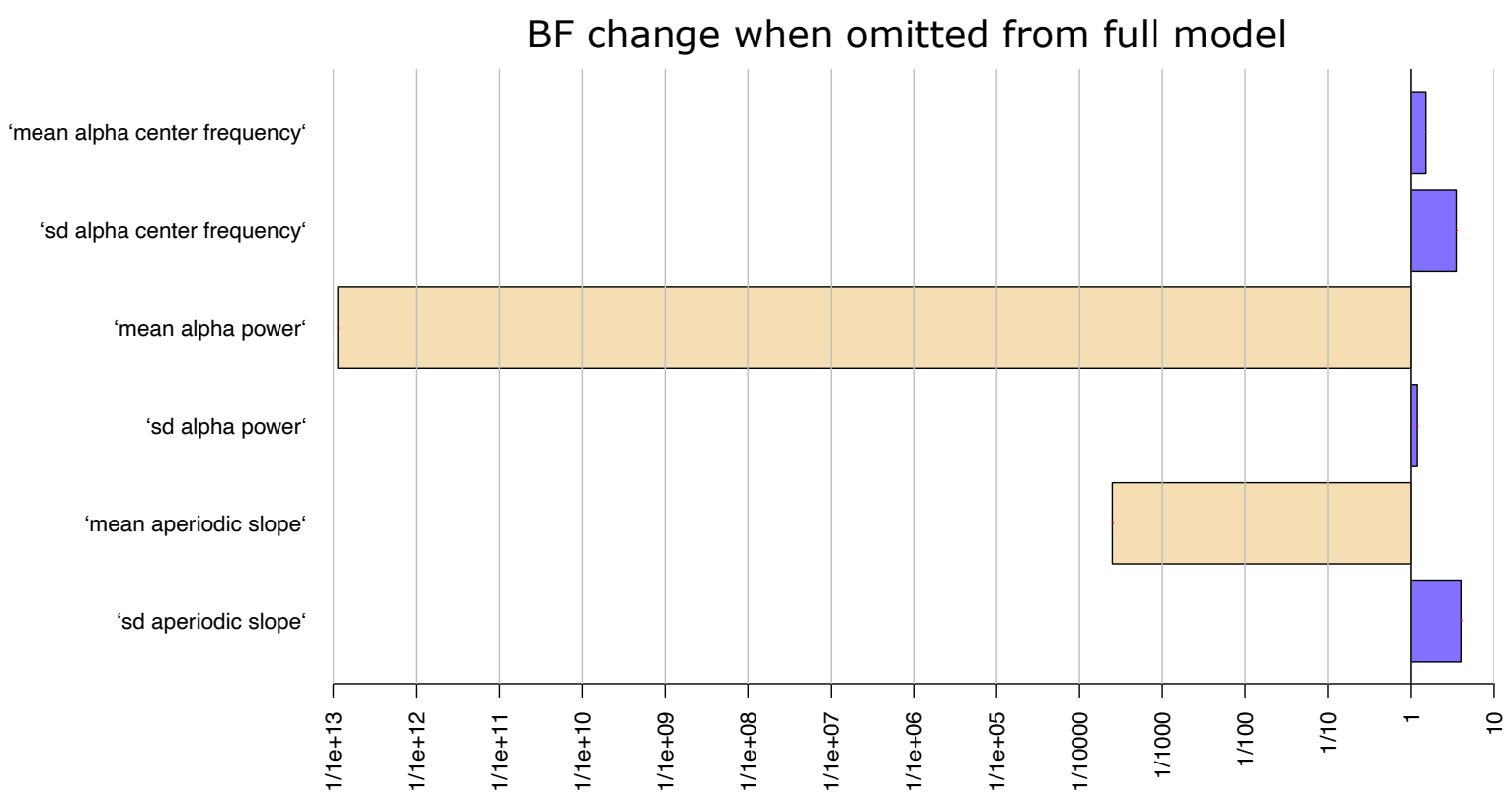

133 Figure 4 - figure supplement 2. Bayes factor analysis for predicting experimental condition

134 (eyes-open vs. eyes-closed). Bayes factor analysis of the linear regression models for predicting

135 condition (eyes -open vs. eyes-closed) from resting-state brain activity. We compared evidence for

136 the full model (see Methods) against reduced models where each predictor was removed

137 sequentially (i.e., SPRiNT parameter). Both the removal of mean alpha power and mean aperiodic

138 slope had substantial negative effect on goodness of fit, with corresponding Bayes factors $<1$,

139 which emphasizes their significance in accounting for the experimental condition (eyes-open vs.

140 eyes-closed). 


\section{Prediction of biological age group from aperiodic and periodic spectral components of}

\section{3 resting-state EEG (empirical data)}

144 We used SPRiNT parameters obtained from both eyes-closed and eyes-open recordings to predict

145 participants' age group (i.e., younger vs. older adults) in the LEMON dataset (Babayan et al.,

146 2019). As discussed in the main text, the mean aperiodic slope and the standard deviation of alpha

147 peak center frequency predicted the participants' age group for eyes-open resting-state recordings

148 (Table 3). A Bayes factor analysis provided strong evidence for mean aperiodic slope and 149 substantial evidence for the standard deviation of alpha peak center frequency (Figure 4 - figure

150 supplement 3). Similarly, we found that mean aperiodic slope and mean alpha center frequency

151 for eyes-closed resting-state recordings predicted the participants' age group (Table 4). A Bayes

152 factor analysis found strong and moderate evidence for mean aperiodic slope and mean alpha

153 center frequency, respectively (Figure 4 - figure supplement 4). Taken together, these results

154 suggest that SPRiNT parameters can predict significant participant demographic features (i.e., the

155 aperiodic slope predicts age group in both the eyes-open and eyes-closed conditions).

156 We also ran the same regression analysis on alpha power extracted from wavelet

157 spectrograms. Mean alpha power (6-14 Hz) predicted age group only in the eyes-closed condition

$158(\beta=-1.14, \mathrm{SE}=0.38,95 \%$ CI $[-1.92,-0.41]$; Table 6; for eyes-open, see Table 5). With SPRiNT,

159 we demonstrated in main text that aperiodic slope and alpha mean center frequency were the

160 strongest predictors of age group. We conclude that the effects identified with the wavelet

161 approach may be confounded by modulations of the aperiodic exponent, or a shift in alpha-peak

162 power outside of a predefined band of interest, as one ages, as discussed by Donoghue et al. (2020)

163 and Scally et al. (2018). 


\begin{tabular}{|c|c|c|c|}
\hline & & Age & \\
\hline Predictors & Log-Odds & $C I$ & $p$ \\
\hline (Intercept) & 1.95 & $-2.91-6.94$ & 0.434 \\
\hline Eyes-open mean alpha center frequency & -0.06 & $-0.41-0.29$ & 0.737 \\
\hline Eyes-open std alpha center frequency & 1.40 & $0.38-2.48$ & 0.008 \\
\hline Eyes-open mean alpha power & 0.31 & $-2.78-3.14$ & 0.834 \\
\hline Eyes-open std alpha power & -3.25 & $-8.49-1.78$ & 0.212 \\
\hline Eyes-open mean aperiodic slope & -3.39 & $-4.95--1.99$ & $<0.001$ \\
\hline Eyes-open std aperiodic slope & 3.65 & $-4.77-11.44$ & 0.370 \\
\hline Observations & 178 & & \\
\hline $\mathrm{R}^{2} \mathrm{Tjur}$ & 0.233 & & \\
\hline
\end{tabular}

165 Table 3. Eyes-open logistic regression model parameters for predicting age group, SPRiNT. 


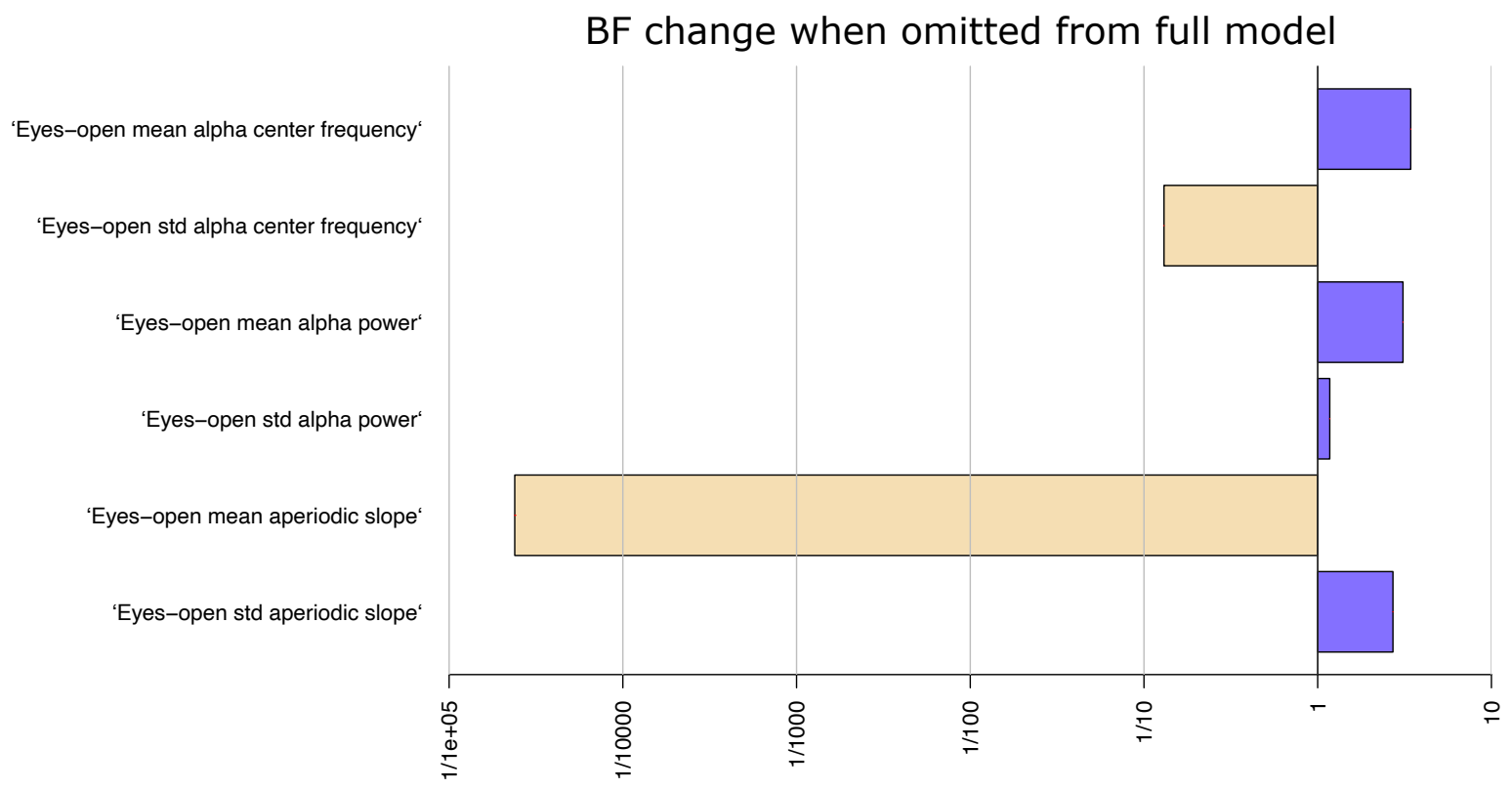

168 Figure 4 - figure supplement 3. Bayes factor analysis for eyes-open resting-state brain

169 activity.

170 Bayes factor analysis for the linear regression models for predicting age group from resting-state

171 eyes-open brain activity. We compared evidence for the full model (see Methods) against reduced

172 models where each predictor was removed sequentially (i.e., SPRiNT parameter). Both the

173 removal of standard deviation of alpha center frequency and mean aperiodic slope had substantial

174 negative effects on the goodness of fit, with corresponding Bayes factors $<1$, which emphasize

175 their significance in accounting for age group.

Predictors

(Intercept) $\log -\mathrm{Odd}$

11.03

$4.44-18.27$ 


\begin{tabular}{lccc} 
Eyes-closed mean center frequency & -0.73 & $-1.27--0.23$ & $\mathbf{0 . 0 0 6}$ \\
Eyes-closed std center frequency & 1.02 & $-0.47-2.56$ & 0.186 \\
Eyes-closed mean alpha power & -0.17 & $-1.81-1.44$ & 0.837 \\
Eyes-closed std alpha power & -0.37 & $-5.23-4.43$ & 0.880 \\
& & & \\
Eyes-closed mean aperiodic slope & -4.32 & $-6.08--2.77$ & $<\mathbf{0 . 0 0 1}$ \\
Eyes-closed std aperiodic slope & 0.83 & $-9.41-9.75$ & 0.863 \\
\hline Observations & 178 & & \\
$\mathrm{R}^{2}$ Tjur & 0.271 & &
\end{tabular}

180 Table 4. Eyes-closed logistic regression model parameters for predicting age group, SPRiNT.

181

BF change when omitted from full model

'Eyes-closed mean alpha center frequency'

'Eyes-closed std alpha center frequency'

'Eyes-closed mean alpha power'

'Eyes-closed std alpha power'

'Eyes-closed mean aperiodic slope

'Eyes-closed std aperiodic slope

\section{3}

184

\section{BF change when omitted from full model}

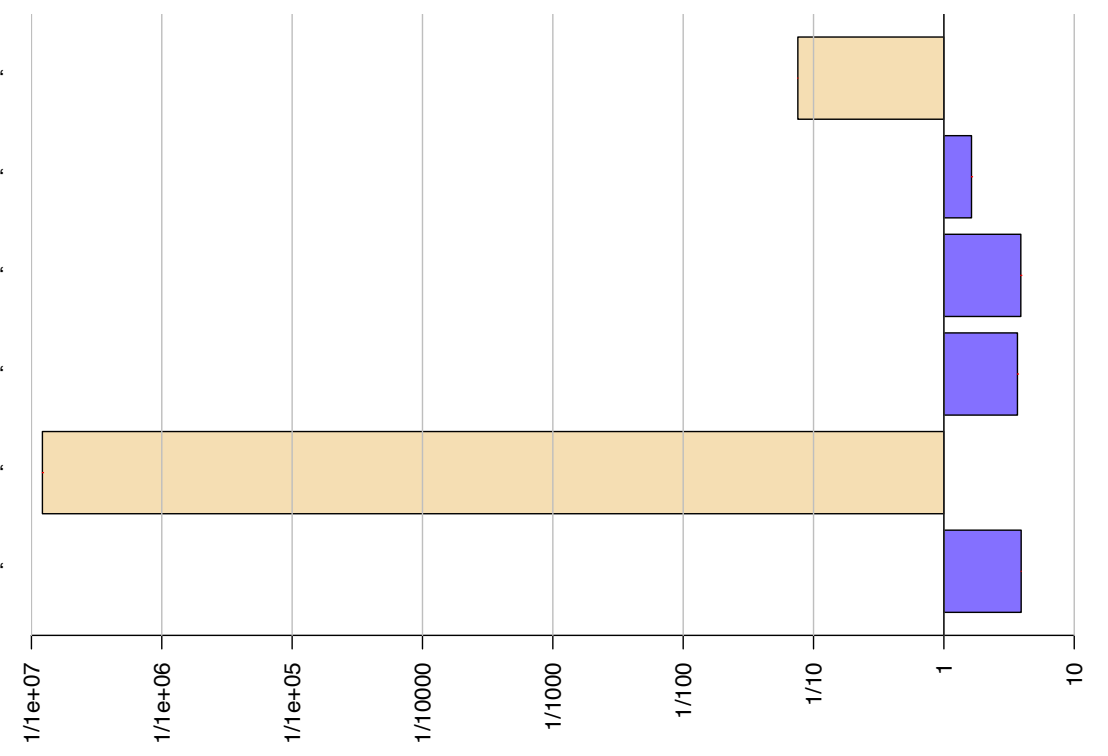

Figure 4 - figure supplement 4. Bayes factor analysis for eyes-closed resting-state brain

activity. Bayes factor analysis for the linear regression models predicting age group from resting- 
185 state eyes-closed brain activity. We compared evidence for the full model (see Methods) against

186 reduced models where each predictor was removed sequentially (i.e., SPRiNT parameter). The

187 removal of mean alpha center frequency and mean aperiodic slope had both substantial negative

188 effects on the goodness of fit, with corresponding Bayes factor $<1$, which emphasize their

189 significance in accounting for age group.

190

191

192

193

194

\begin{tabular}{lccc}
\hline \hline & & Age & \\
& & & \\
Predictors & Log-Odds & $C I$ & $p$ \\
\hline (Intercept) & -9.48 & $-22.17-2.67$ & 0.133 \\
& & & \\
Eyes-open alpha power (Morlet wavelets) & -0.68 & $-1.67-0.27$ & 0.166 \\
& & & \\
\hline Observations & 178 & & \\
$\mathrm{R}^{2}$ Tjur & & &
\end{tabular}

195 Table 5. Eyes-open logistic regression model parameters for predicting age group, Morlet

196 wavelets $(\mathrm{BF}=2.50)$.

\begin{tabular}{llcl}
\hline \hline & Age & \\
& & & \\
Predictors & Log-Odds & $C I$ & $p$ \\
& & & \\
\hline
\end{tabular}




\begin{tabular}{llll} 
(Intercept) & -15.02 & $-24.83--5.93$ & $\mathbf{0 . 0 0 2}$ \\
Eyes-closed alpha power (Morlet wavelets) & -1.14 & $-1.92--0.41$ & $\mathbf{0 . 0 0 3}$ \\
& & & \\
\hline Observations & 178 & \\
& & \\
$\mathrm{R}^{2}$ Tjur & 0.053 &
\end{tabular}

198 Table 6. Eyes-closed logistic regression model parameters for predicting age group, Morlet

199 wavelet spectrogram $(\mathrm{BF}=0.07)$.

a) subject EC012

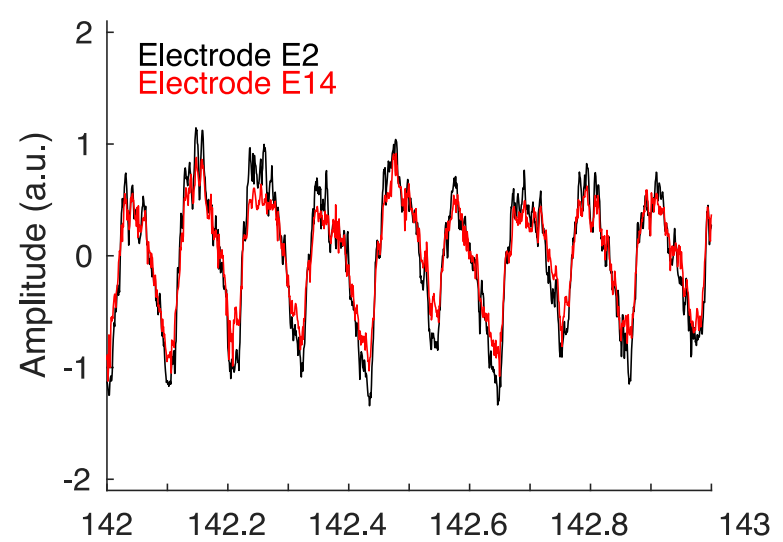

200 b) subject EC013

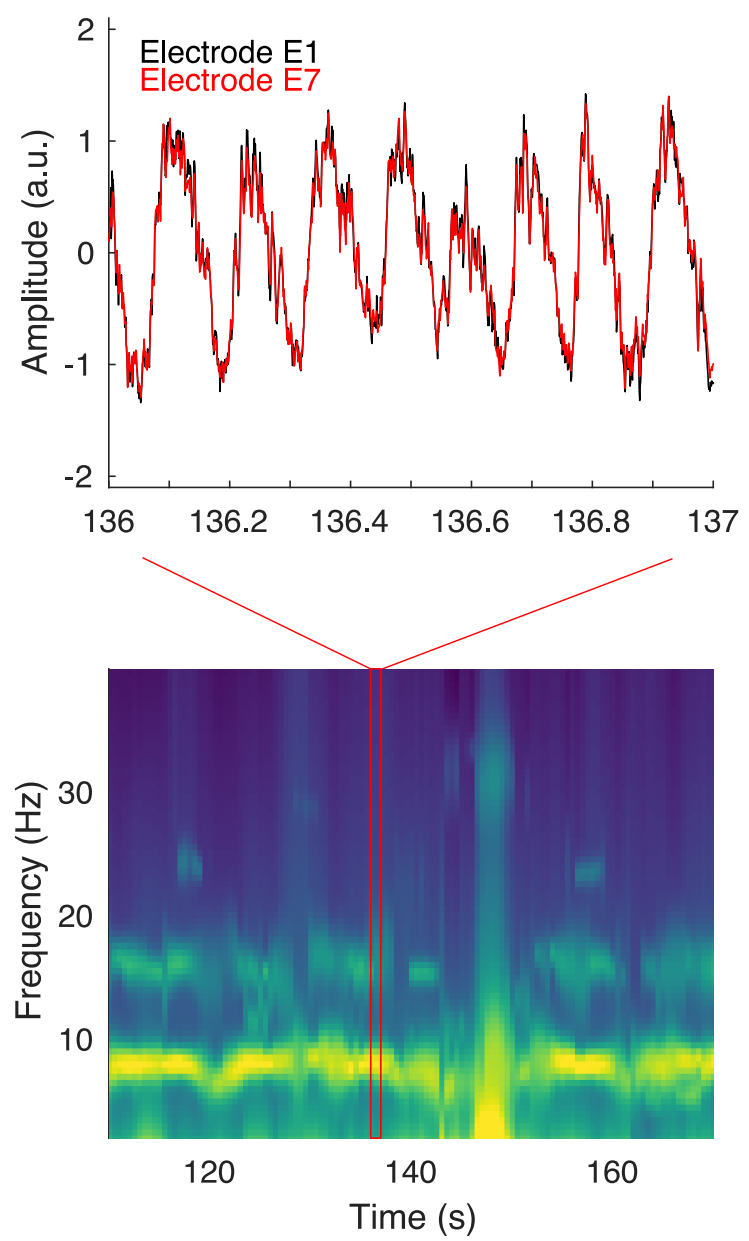

Figure 5 - figure supplement 1. Examples of sawtooth rhythms from two representative

electrodes in entorhinal cortex layer 3 from both subjects. (A) Example time-series of 
203 prominent sawtooth rhythms from two representative electrodes during a movement bout for

204 subject EC012 (top), producing harmonics of activity in the average modelled spectrogram

205 (bottom). (B) Same as (A), for subject EC013.

a) subject EC012

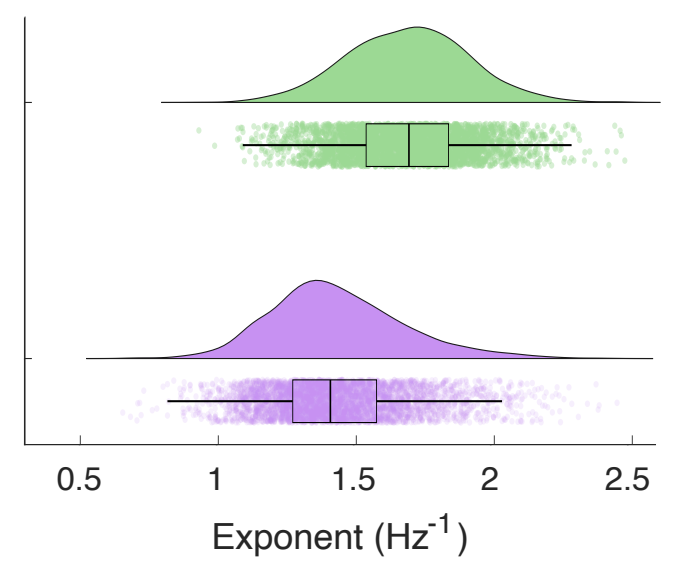

b) subject EC013

206

207

208

209

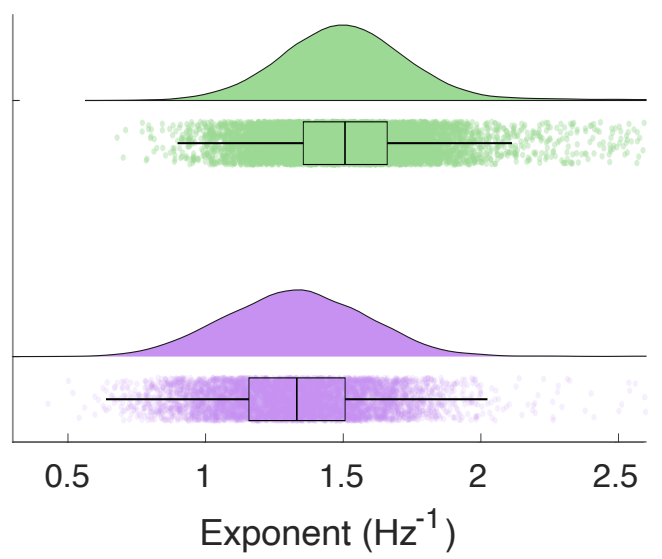

rest

movement
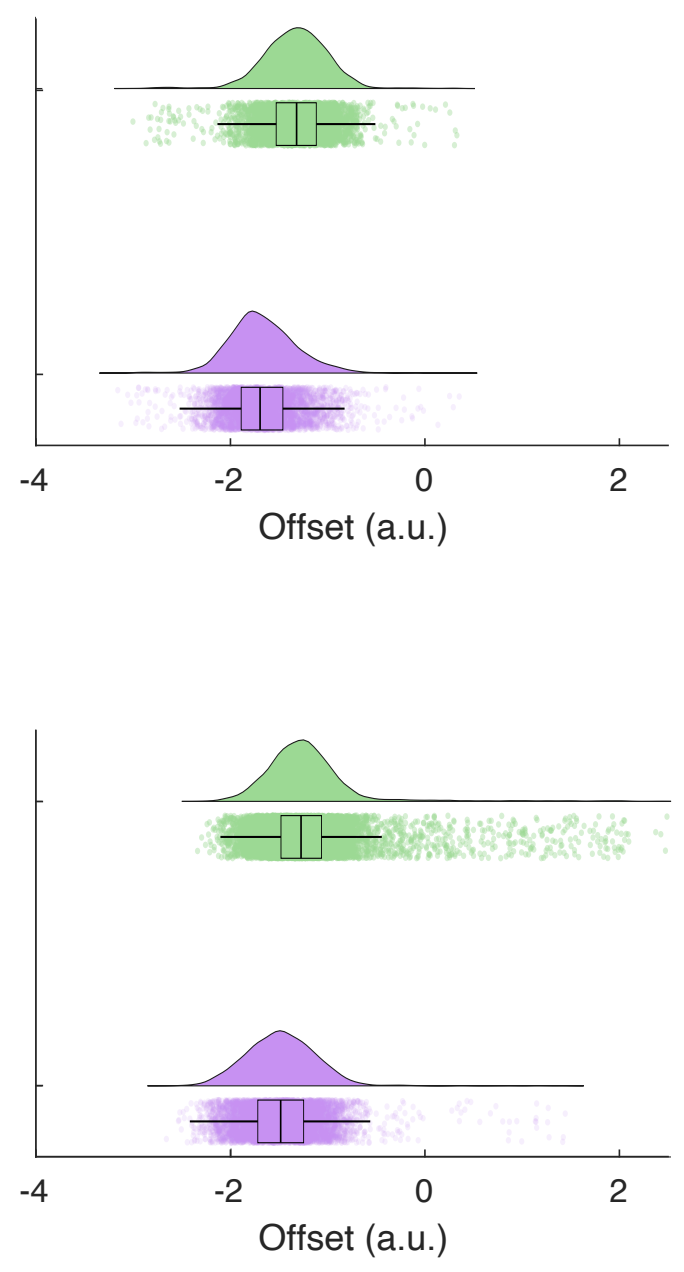

Figure 5 - figure supplement 2. Empirical distributions of SPRiNT aperiodic exponent and

offset parameters. (A) Empirical distributions of aperiodic exponent and offset estimates for subject EC012 at time bins associated with rest (green) and movement (purple) bouts (boxplot

210 line: median; boxplot limits: first and third quartiles; whiskers: range). (B) Same as (A), for subject 
211 EC013. Sample sizes: EC012 rest (movement) = 3,896 (4,584) bins; EC013 rest (movement) = $21220,970(10,386)$ bins.

\section{References}

217 Babayan, A., Erbey, M., Kumral, D., Reinelt, J. D., Reiter, A. M. F., Röbbig, J., .. . Villringer, physiology in young and old adults. Scientific Data, 6(1), 180308.

221 Donoghue, T., Haller, M., Peterson, E. J., Varma, P., Sebastian, P., Gao, R., .. Knight, R. T. (2020).

$$
\text { Neuroscience, 23(12), 1655-1665. doi:10.1038/s41593-020-00744-x }
$$

224 Donoghue, T., Schaworonkow, N., \& Voytek, B. (2021). Methodological considerations for studying neural oscillations. Eur J Neurosci. doi:10.1111/ejn.15361

226 Scally, B., Burke, M. R., Bunce, D., \& Delvenne, J.-F. (2018). Resting-state EEG power and 227 connectivity are associated with alpha peak frequency slowing in healthy aging. Neurobiology of Aging, 71, 149-155. doi:10.1016/j.neurobiolaging.2018.07.004 